\title{
Hypergeometric Functions: From One Scalar Variable to Several Matrix Arguments, in Statistics and Beyond*
}

\author{
T. Pham-Gia ${ }^{1,2}$, Dinh Ngoc Thanh ${ }^{2,3}$ \\ ${ }^{1}$ Department of Mathematics and Statistics, Université de Moncton, Moncton, Canada \\ ${ }^{2}$ The Applied Multivariate Research Group, Université de Moncton, Moncton, Canada \\ ${ }^{3}$ Department of Mathematics and Computer Science, University of Science, Vietnam National University, Ho Chi Minh City, Vietnam \\ Email: thu.pham-gia@umoncton.ca,dnthanh@hcmus.edu.vn
}

How to cite this paper: Pham-Gia, T. and Thanh, D.N. (2016) Hypergeometric Functions: From One Scalar Variable to Several Matrix Arguments, in Statistics and Beyond. Open Journal of Statistics, 6, 951-994. http://dx.doi.org/10.4236/ojs.2016.65078

Received: July 18, 2016

Accepted: October 24, 2016

Published: October 27, 2016

Copyright $\odot 2016$ by authors and Scientific Research Publishing Inc. This work is licensed under the Creative Commons Attribution International License (CC BY 4.0).

http://creativecommons.org/licenses/by/4.0/

\begin{abstract}
Hypergeometric functions have been increasingly present in several disciplines including Statistics, but there is much confusion on their proper uses, as well as on their existence and domain of definition. In this article, we try to clarify several points and give a general overview of the topic, going from the univariate case to the matrix case, in one and then in several arguments. We also survey some results in fields close to Statistics, where hypergeometric functions are actively used, studied and developed.
\end{abstract}

\section{Keywords}

Hypergeometric, Zonal Polynomial, Fractional Calculus, Lie Group, Cohomology, Computation

\section{(c) (†) Open Access}

\section{Introduction}

Hypergeometric functions in one or several variables, introduced first in Mathematics, have been used in Physics and Applied Mathematics for some time. But their presence in Statistics is quite recent, within various topics, particularly in operations on random variables and on non-null distributions. In Multivariate analysis, as reported by Bose [1], Gauss hypergeometric function was used by Fisher as early as in 1928, in the determination of the density of the sample multiple correlation coefficient $R^{2}$.

There is, however, some confusion regarding the different forms under which the hypergeometric function appears. In particular, the equalities between the infinite series, the Euler integral representation, the Laplace representation and the Mellin-Barnes 
representation can be confusing. Since they are only valid under certain conditions, one form can converge while the others do not, or take different values. We will discuss the necessary conditions for their equivalences, for ${ }_{2} F_{1}($.$) only, but similar considerations$ hold for ${ }_{p} F_{q}($.$) . We will show the progression of these notions, from the scalar case to$ the matrix case.

In this article, we are mostly concerned with the presence of hypergeometric functions in Statistics and to this end, have adopted two measures: Section 7 is completely devoted to Statistics, and in the last part of the article we will survey hypergeometric functions in various domains, and discuss their potential relations with, and applications in, Statistics. Throughout the text, whenever possible, we will also express similar opinions, which are strictly ours, and are necessarily subjective.

We try to be informative without being too technical. Naturally, we can only give a general landscape on the hypergeometric functions' presence in neighboring fields. We will not go into details when coming into a specific domain, since this would require advanced knowledge in that domain itself. But relevant references are given so that the reader can deepen his/her knowledge on a certain topic if she/he so wishes. We have also given up the effort of trying to present a unified set of notations/symbols throughout the paper because these notions vary so much from one field to the other. We believe that a good grasp of the whole picture will allow readers to have an appreciation of the diversity and richness of hypergeometric functions. Then, they can make possible connections between these ideas and their own statistical domain, to derive other results and conclusions.

There are, at present, three survey articles on hypergeometric functions in the literature: one is from the Encyclopedia of Statistical sciences [2], one by Schlosser [3], and the third one by Abadir [4]. Each of these surveys has its own merits, but the first one is limited to one variable and does not cover several topics related to mathematics. The second one is strictly mathematical and covers multivariate series only, while the last one is oriented toward economics/econometrics topics. Furthermore, there are a couple of surveys in Wikipedia [5], which are also quite informative, and a short article in Encyclopedia of Mathematics (Russian [6]). The present article hopes to complement all these surveys and studies.

Leon Ehrenpreis [7] wrote: "Hypergeometric functions pervade many branches of mathematics because it is at the confluence of three fundamental viewpoints." And Cattani [8] reported that in the MathSciNet data base there were already 3181 articles with title word hypergeometric, of which 1530 were published since 1990. At present, there are several distinct topics in the mathematical/statistical literatures related to the word Hypergeometric, such as hypergeometric integrals, hypergeometric groups, etc. beside more specific terms like hypergeometric polynomials, rational hypergeometric functions, etc.

In the same spirit, Askey [9] wrote in his review of Carlson's [10] book. "At present no one has a good overview of what is happening to multivariate extensions of hypergeometric functions", and predicted that full comprehension of multiple hypergeome- 
tric series will take another hundred years. But, fortunately, Gelfand, Kapranov and Zevelinsky [11] have already given a partial reaction to this statement. On the other hand, Saito, Sturmfels and Nakayama [12] have mentioned the problem that hypergeometric functions and series have been lately treated from so many points of view completely different from each other. Here, we will attempt to connect some of them to Statistics, and, in the process, will evidence three themes:

1) The versatility of hypergeometric functions is due to the fact that they can be expressed as an infinite series, or as very different forms of integrals. The three basic forms, Euler, Laplace and Mellin-Barnes, can then be studied and extended, using mathematical analysis tools.

2) Some common approaches used by researchers are: averaging (through different processes) and progressive definitions (e.g. from $(p, q)$ to $(p+1, q)$, starting or finishing at simple common functions.

3) In Statistics, understandably, Hypergeometric functions are not developed, but used, mostly in distribution theory. However, James [13] and Constantine and Muirhead [14] have contributed significantly to the theory of zonal polynomials.

In section 2 we will consider the univariate scalar case and progressive generalizations of the hypergeometric functions, from three parameters to n parameters and to $H$ and $G$-functions. Since integral representations play a key role, we have presented them clearly at every step. In Section 3, we generalize to several scalar variables, again giving the three integral representations. In Section 4, we consider one or several matrix variates and the three current approaches to introduce them. In Section 5, computational issues will be discussed. Section 6 gives some other approaches used to derive the hypergeometric functions, different from the classical one. In Section 7, the presence of hypergeometric functions in Statistics, will be presented, with no pretention of being exhaustive. Finally, in section 8 we present the hypergeometric function in neighboring domains, with potential connections to Statistics. Since there are so many such domains, we do not pretend to be exhaustive, or objective here either, and can only give basic ideas of interest, or results of importance. Deeper results would, naturally, require specialized advanced technical knowledge from the reader in that domain.

NOTE: In this survey we will limit our consideration to the real case, for scalar, vector and matrix variables, since the complex case is seldom encountered in Statistics, and its inclusion would considerably lengthen the article. Classical treatises on this topic are Erdelyi et al. [15], Slater [16] [17], Bailey [18]. They are excellent references that we wish to acknowledge here, but there are certainly others that we ignore, and we would appreciate having them brought to our attention. Also, articles from various contributors mentioned here have been chosen to illustrate various points presented in the article, and not because they are the most influential, or the most important.

We also realize that to cover such an immense topic as hypergeometric functions, within a limited number of pages, our survey is very ambitious and necessarily incomplete in many respects. Several properties of Gauss hypergeometric function related to continued fractions, linear and quadratic transformations, etc., could not be treated due 
to lack of space. We hence ask for your comprehension and understanding.

To put more clarity into our presentation we have worked out the following plan, which also reflects our point of view on surveying the whole topic: integral representations within progressive generalizations. Naturally, our view is only one among so many others, that could differ sharply from ours.

\section{PLAN OF THE PRESENTATION}

1. Introduction

2. Hypergeometric series and functions in one scalar variable

2.1. The Laplace, Fourier and Mellin Transforms

2.2. Sums versus integrals

2.3. Integral representations

2.3.1. Euler integral on a finite segment of the real line

2.3.2. Laplace representation on the positive half-line

2.3.3. Mellin-Barnes representation by contour integral in the complex plane

2.3.4. Contiguous relations

2.4. Generalization to several parameters

2.4.1. Generalized hypergeometric functions

2.4.2. Analytic continuation

2.4.3. Euler integral representation

2.4.4. Laplace representation on the positive axis

2.4.5. Mellin-Barnes representation

2.5. Generalization to $\mathrm{G}$ and $\mathrm{H}$ - functions

3. Hypergeometric series and functions in several independent scalar variables

3.1. Appell, Lauricella and others sums

3.2. Integral representations and further generalization

3.2.1. Integral representation of Euler type

3.2.2. Integral representation of Laplace type on $(0, \infty)^{n}$

3.2.3. Representation of Mellin-Barnes type

3.3. Differential Equations and systems

3.4. Generalized $\mathrm{G}$ and $\mathrm{H}$-functions in several independent scalar variables

4. Hypergeometric functions in matrix arguments: three proposed approaches

4.1. Functions in one matrix variate

4.1.1. Laplace transform approach

4.1.2. Zonal Polynomials approach

4.1.3. Matrix-transforms approach

4.2. Hypergeometric function in two matrix variates

5. Computational Issues

5.1. Computation of the hypergeometric function

5.2. Old and new relations between hypergeometric functions managed by computer

6. Hypergeometric functions derived via other approaches

6.1. Fractional Calculus 


\subsection{Lie Group approach}

6.3. Carlson's approach

6.3.1. Definitions of functions $R_{n}(b, z)$ and $S(b, z)$ as averages

6.3.2. Results of interest

6.3.3. Single integral representation and Elliptic integrals

6.4. Basic q-hypergeometric functions

7. Presence of Hypergeometric Functions in Statistics

7.1. Discrete case

7.2. Continuous case

7.3. Matrix case

7.4. Other Applications

8. Hypergeometric Functions in Neighboring Domains

8.1. Algebraic topology, Algebraic K-Theory, Algebraic Geometry

8.1.1. Integral representations

8.1.2. Single Integral representation

8.1.3. A-Hypergeometric functions

8.2. Hypergeometric integrals in Conformal Field theory, Homology and Cohomology

8.3. Algebraic functions and roots of equations

8.4. Economics, Quantitative Economics and Econometrics

8.5. Random matrices in Theoretical Physics

9. Conclusion

10. References

End

\section{Hypergeometric Series and Functions in One Scalar Variable}

\subsection{The Laplace, Fourier and Mellin Transforms}

These three transforms play key roles in this article:

a) For a function $f(x)$ such that $\int_{0}^{\infty} \mathrm{e}^{-k x}|f(x)| \mathrm{d} x<\infty$ for some real value $k$, the Laplace transform of $f(x), x \geq 0$, is

$$
L_{t}\{f(x)\}=L_{f}(t)=\int_{0}^{\infty} \mathrm{e}^{-r x} f(x) \mathrm{d} x,
$$

where $r$ is a complex variable. Conversely, if $L_{t}\{f(x)\}$ is analytic, of order $O\left(r^{-k}\right)$ in some half-plane $\operatorname{Re}(r)>c$, with $c, k$ real and $k>1$, then its inverse is $f(x)$, uniquely determined by:

$$
f(x)=\frac{1}{2 i \pi} \lim _{\beta \rightarrow \infty} \int_{w-i \beta}^{w+i \beta} \mathrm{e}^{t x} L_{f}(t) \mathrm{d} t, \quad t \in C,
$$

evaluated over any line $\operatorname{Re}(r)=w>c$ in the complex plane.

Two functions with same Laplace transform are identical. If $f(x)$ is the density of 
$X$

$$
\theta_{t}\{f(x)\}=\int_{0}^{\infty} \mathrm{e}^{t x} f(x) \mathrm{d} x
$$

is the moment generating function of $X$.

b) The Fourier transform of $f(x),-\infty<x<\infty$, s.t. $\int_{-\infty}^{\infty}|f(x)| \mathrm{d} x<\infty$, for some real $k$ is:

$$
F_{t}\{f(x)\}=F_{f}(t)=\int_{-\infty}^{\infty} \mathrm{e}^{-i t x} f(x) \mathrm{d} x
$$

and its inverse is

$$
f(x)=\frac{1}{2 \pi} \int_{-\infty}^{\infty} \mathrm{e}^{i x t} F_{f}(t) \mathrm{d} t
$$

c) The Mellin transform of $f(x), \quad x \geq 0$, where $\int_{0}^{\infty} x^{k-1}|f(x)| \mathrm{d} x<\infty$ for some real $k$, is defined by:

$$
\mathbf{M}_{s}\{f(x)\}=\int_{0}^{\infty} x^{s-1} f(x) \mathrm{d} x .
$$

Then its inverse Mellin transform is:

$$
f(x)=M_{x}^{-1}\left[\mathbf{M}_{s}(f(x))\right]=\frac{1}{2 i \pi} \int_{w-i \infty}^{w+\infty \infty} x^{-s} \mathbf{M}_{s}(f(x)) \mathrm{d} s, s \in \mathbb{C} .
$$

Equation (3) is valid under the condition that (2) exists as an analytic function of the complex variable $s$, for $c_{1} \leq \operatorname{Re}(s)=w \leq c_{2}$. The integral is independent of $w$.

\subsection{Sums Versus Integrals}

In this section we consider only series and their limits. We have the series representation of the exponential function, which is a special case of the hypergeometric series:

$$
\exp (x)=\sum_{n=0}^{\infty} \frac{x^{n}}{n !}, \quad x \in \mathbb{C},
$$

where the ratio of two consecutive coefficients:

$$
\frac{a_{n+1}}{a_{n}}=\frac{1}{n+1}, \quad a_{j} \in \mathbb{R}^{+} .
$$

One generalization of this notion is associated with the hypergeometric series, where this ratio is a rational expression of $n$. Then we should have:

$$
\frac{a_{n+1}}{a_{n}}=\frac{r\left(a_{1}+n\right) \cdots\left(a_{p}+n\right)}{s\left(b_{1}+n\right) \cdots\left(b_{q}+n\right)} \cdot \frac{1}{(1+n)}
$$

in its decomposition into a rational form, i.e. depending on $p+q$ constants and 2 other constants $r$ and $s$.

The corresponding series is then, 


$$
=1+\frac{a_{1} \cdots a_{p}}{b_{1} \cdots b_{q}} \frac{1}{1}\left(\frac{r z}{s}\right)+\frac{a_{1} \cdots a_{p}}{b_{1} \cdots b_{q}} \frac{\left(a_{1}+1\right) \cdots\left(a_{p}+1\right)}{\left(b_{1}+1\right) \cdots\left(b_{q}+1\right)} \cdot \frac{1}{2}\left(\frac{r z}{s}\right)^{2}+\cdots
$$

which becomes, after rearranging and change of scale:

$$
=1+\frac{a_{1} \cdots a_{p}}{b_{1} \cdots b_{q}} \frac{z}{1 !}+\frac{a_{1}\left(a_{1}+1\right) \cdots a_{p}\left(a_{p}+1\right)}{b_{1}\left(b_{1}+1\right) \cdots b_{q}\left(b_{q}+1\right)} \frac{z^{2}}{2 !}+\cdots
$$

The hypergeometric series $H S_{(p, q)}\left(a_{1}, \cdots, a_{p} ; b_{1}, \cdots, b_{q} ; z\right)$ has the above expression. For $p=2, q=1$, we have Gauss hypergeometric series in 3 parameters

$$
H S_{(2,1)}(a, b ; c ; z)=\sum_{n=0}^{\infty} \frac{(a)_{n}(b)_{n}}{(c)_{n}} \frac{z^{n}}{n !}, \quad z \in \mathbb{C},
$$

where the Pochhammer symbol is $(a)_{n}=a(a+1) \cdots(a+n-1),(a)_{0}=1$. Equation (4) reduces to the geometric series for $a=b=c=1$, hence its name. $a$ and $b$ can be any real or complex value but $c$ must be different from a negative integer. If $a$ or $b$ is zero or a negative integer the series becomes a polynomial.

The first work on hypergeometric function was made by Euler in 1687, when he studied series (4), as solution to Equation (21). Gauss (1812) and Riemann (1857) continued Euler's work in the complex domain and solved the associated multivaluedness problem, presently known as monodromy problem.

\subsection{Integral Representations}

The whole field of Special Functions is characterized by integral representations of various kinds (see e.g. Lebedev [19]). We first recall the integral representation of the upper tail of the gamma distribution by a finite sum, well known in elementary statistics (Hogg and Craig [20], p. 132):

$$
\int_{\lambda w}^{\infty} \frac{z^{k-1} \mathrm{e}^{-z}}{(k-1) !} \mathrm{d} z=\sum_{x=0}^{k-1} \frac{(\lambda w)^{x} \mathrm{e}^{-\lambda w}}{x !}, k \in \mathbb{N}^{+} .
$$

Similarly, we have the integral representation of an infinite series. There are several advantages in dealing with an integral instead of a series, as already remarked by Carlson [10]. Continuity and even analyticity are usually provided by the integral, hence leading to a deeper study of its properties and extensions, and also faster convergence on a digital computer. The hypergeometric series (4), with its convergence region will be of limited interest if it cannot be extended to the whole complex plane. The principle of analytic continuation in complex analysis will permit us to precisely do that operation.

There are three integral representations of $H_{(2,1)}(a, b ; c ; z)$, of increasing complexity, that serve three different purposes, and propose three different ways in computing the values of a hypergeometric function:

\subsubsection{Euler Integral on a Finite Segment of the Real Line} Let 


$$
I_{1}(z)=\frac{\Gamma(c)}{\Gamma(b) \Gamma(c-b)} \int_{0}^{1} t^{b-1}(1-t)^{c-b-1}(1-t z)^{-a} \mathrm{~d} t
$$

and

$$
I_{2}(z)=\frac{\Gamma(c)}{\Gamma(a) \Gamma(c-a)} \int_{0}^{1} t^{a-1}(1-t)^{c-a-1}(1-t z)^{-b} \mathrm{~d} t .
$$

For $a, b, c$ real, inside the unit disc $|z|<1$ we have $H S_{(2,1)}(a, b ; c ; z)=I_{1}(z)$ if $c>c-b>0$ and $H S_{(2,1)}(a, b ; c ; z)=I_{2}(z)$ if $c>c-a>0$. If both double conditions are satisfied then $H S_{(2,1)}(a, b ; c ; z)=I_{1}(z)=I_{2}(z)$.

Outside the unit disc $|z|<1$, either integral can be seen to converge for any value of $z$, except on $[1, \infty)$, for $0<\operatorname{Re}(b)<\operatorname{Re}(c-b)$ or $0<\operatorname{Re}(b)<\operatorname{Re}(c-a)$, respectively. Hence, the condition $|z|<1$ can be dropped and the series can be extended to a function analytic in the complex plane, with a cut along $[1, \infty)$ if $\operatorname{Re}(c)>\operatorname{Re}(b)$ or $\operatorname{Re}(a)$ (Lebedev [19]). It serves to generalize the series $H S_{(2,1)}(a, b ; c ; z)$ outside the unit circle, by analytic continuation. This is the representation which is mostly used in statistics, where, frequently, the integral is encountered first, and hence the series can become redundant.

But the terminology can become confusing. ${ }_{2} F_{1}(a, b ; c ; z) \equiv H S_{(2,1)}(a, b ; c ; z)$ now means the function defined by this integral on the half-line $(-\infty, 1]$ (and on all the complex plane cut along $[1, \infty)$ if $z$ is complex), with an alternate expression as an infinite series within the unit disk, as already suggested by Appell and Kampé de Fériet in 1926 [21]. Also, these integrals are not defined for real positive values of $z$ superior to 1 , as the cut $[1, \infty)$ implies, but they converge for all complex values of $a, b$ and $c$, and are analytic functions of these parameters for $z$ fixed.

\section{Example 1:}

a) Using MAPLE, with $a=4.2, b=3.7, c=6.5$, we have $I_{1}(0.75)=I_{2}(0.75)=H S_{(2,1)}(0.75)=0.002498$ but $I_{1}(2.75), \quad I_{2}(2.75)$, and $H S_{(2,1)}(2.75)$ are non-existent, in good accordance with the theory, while

$$
I_{1}(-12.0)=I_{2}(-12.0)=H S_{(2,1)}(-12.0)=0.000804,
$$

the last value being, however, taken (arbitrarily) from $I_{1}$ by analytic continuation, since we know that the series $H S_{(2,1)}($.$) diverges at -12.0$. Also,

$\lim _{z \rightarrow 1^{-}} F_{1}(a, b ; c ; z)=\frac{\Gamma(c) \Gamma(c-a-b)}{\Gamma(c-a) \Gamma(c-b)}$ if $c-a-b>0$, which is NOT the case here and the limit is $+\infty$.

b) For $a=4.2, b=-3.7, c=8.5$, we have similar results, and equality of $I_{2}(z)$ and $H_{(2,1)}(z)$ on $(-\infty, 1)$, while $I_{1}(z)$ is not defined anywhere on $(-\infty, \infty)$. Figure 1 and Figure 2 illustrate the example.

These integral representations (5) and (5') are very convenient because even when $a$, $b$ and $c$ differ by integers, thi(e)s(e) integral(s) still converge(s), and equal(s) the series within the convergence domain of the latter. This is to be compared with the MellinBarnes representation in 2.3.3 where the poles must be simple, which does not happen when $a, b$ and $c$ differ by integers. 


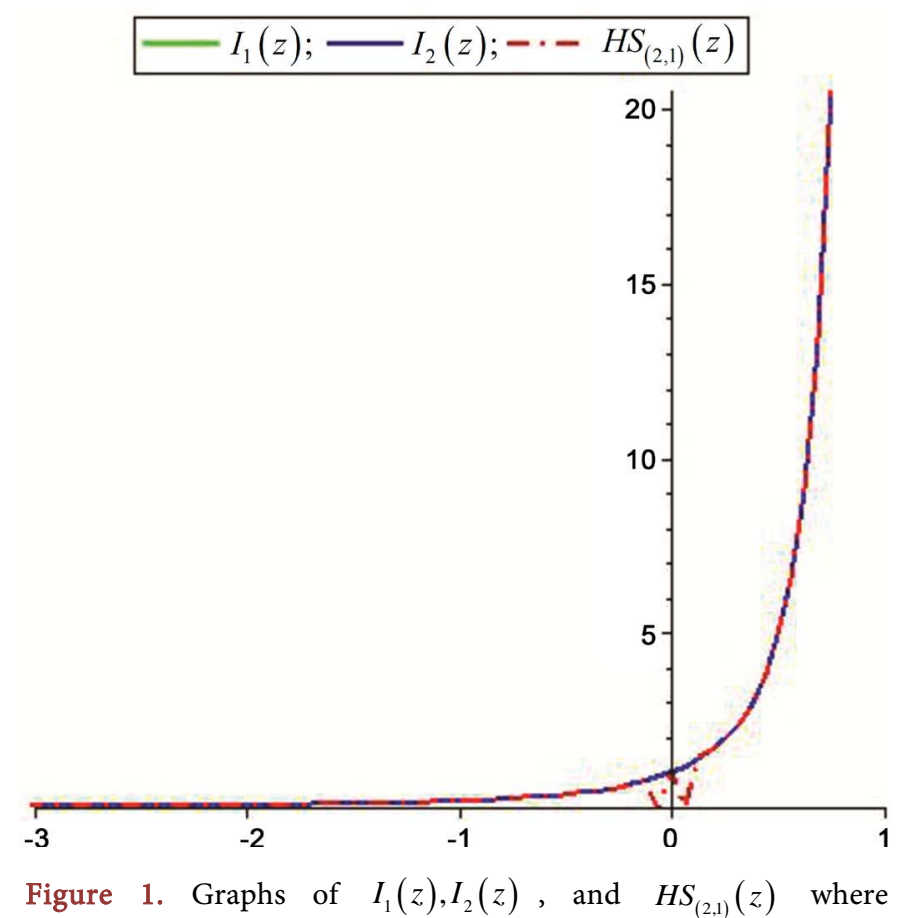

$a=4.2, b=3.7, c=6.5$ (They coincide).

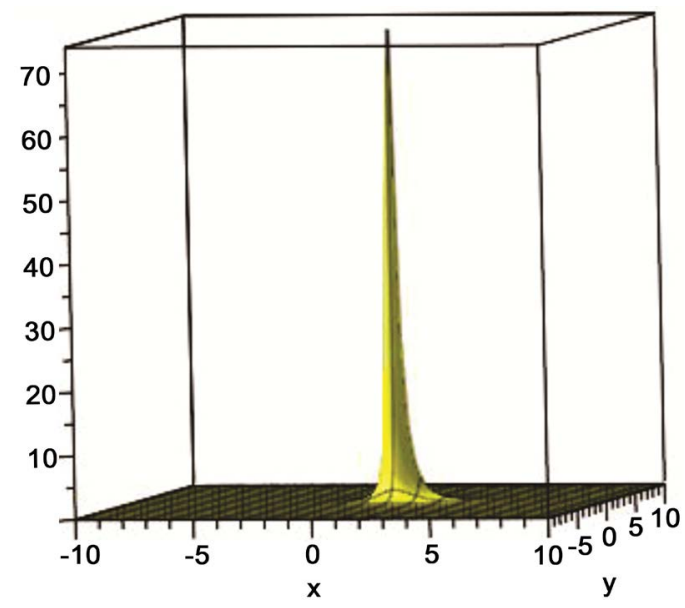

Figure 2. Graphs of $\left|I_{1}(z)\right|$ (a), $\left|I_{2}(z)\right|$ (b), and $\left|H S_{(2,1)}(z)\right|$ (c), with $z=x+i y, a=4.2, b=3.7, c=6.5$ (They coincide).

\subsubsection{Laplace Representation on the Positive Half-Line}

This representation is useful when dealing with Laplace transform methods and moment generating functions, which is frequent in Statistics. However, ${ }_{2} F_{1}($.$) , and later$ ${ }_{p} F_{q}($.$) , is usually expressed in function of another hypergeometric function, with less$ parameters, and this fact is useful for a progressive definition of a family of functions. We have:

$$
{ }_{2} F_{1}(a, b ; c ; z)=\frac{1}{\Gamma(a)} \int_{0}^{\infty} \mathrm{e}^{-t} t^{a-1} \cdot{ }_{1} F_{1}(b, c ; z t) \mathrm{d} t, \quad \operatorname{Re}(a)>0,
$$


where ${ }_{1} F_{1}(\cdot)$ is the confluent hypergeometric function, studied first by Kummer [22], with single integral representation:

$$
{ }_{1} F_{1}(a, c ; z)=\sum_{k=0}^{\infty} \frac{(a)_{k}}{(c)_{k}} \frac{z^{k}}{k !}=\frac{\Gamma(c)}{\Gamma(a) \Gamma(c-a)} \int_{0}^{1} \mathrm{e}^{z t} t^{a-1}(1-t)^{c-a-1} \mathrm{~d} t, \quad \operatorname{Re}(c-a)>0 .
$$

or double integral representation:

$$
\frac{1}{\Gamma(a) \Gamma(b)} \int_{0}^{\infty} \int_{0}^{\infty} \mathrm{e}^{-t-s} s^{a-1} t^{b-1} \cdot{ }_{0} F_{1}(-; c ; x s t) \mathrm{d} s \mathrm{~d} t .
$$

This hypergeometric function is an important function in its own right (see Slater [16]), but due to space limitation we will not deal with it further. On the other hand, the Laplace transform of ${ }_{p} F_{q}($.$) is:$

$\int_{0}^{\infty} \mathrm{e}^{-t x} t_{p}^{\alpha-1} F_{q}\left(a_{1}, \cdots, a_{p} ; b_{1}, \cdots, b_{q} ; t\right) \mathrm{d} t=\frac{\Gamma(\alpha)}{x^{c}}{ }_{p+1} F_{q}\left(a_{1}, \cdots, a_{p}, \alpha ; b_{1}, \cdots, b_{q} ; x^{-1}\right)$, (see (10)) which, however, is valid only for $p<q, \operatorname{Re}(\alpha)>0, \operatorname{Re}(x)>0$, or $p=q$, $\operatorname{Re}(\alpha)>0$, and does not apply here.

MATHEMATICA gives this transform a quite complex sum of three hypergeometric functions, as follows:

$$
\begin{aligned}
L\left\{{ }_{2} F_{1}(a, b ; c ;-t)\right\}(z)= & \frac{\Gamma(1-a) \Gamma(b-a) \Gamma(c) z^{a-1}}{\Gamma(b) \Gamma(c-a)} \cdot{ }_{1} F_{1}(a-c+1, a-b+1 ; z) \\
& +\frac{\Gamma(1-b) \Gamma(a-b) \Gamma(c) z^{b-1}}{\Gamma(a) \Gamma(c-b)} \cdot{ }_{1} F_{1}(b-c+1,1-a+b ; z) \\
& +\frac{c-1}{(a-1)(b-1)} \cdot{ }_{2} F_{2}(1,2-c ; 2-a, 2-b ; z), \quad \operatorname{Re}(z)>0 .
\end{aligned}
$$

NOTE: Some results on this transform, and its inverse, are given on p.212 and 291 of Tables of Integral Transforms [15].

\subsubsection{Mellin-Barnes Representation by Contour Integral in the Complex Plane}

Complex analysis developed in the 19th century brought powerful tools such as the calculus of residues, and Mellin-Barnes formula gives a third representation, based on contour integration. The value of the integral is computed, not as a complex integral, but as the sum of the residues at poles of $\Gamma(s)$. When they are simple we have:

$$
H S_{(2,1)}(a, b ; c ; z)=\frac{1}{2 \pi i} \frac{\Gamma(c)}{\Gamma(a) \Gamma(b)} \int_{-i \infty}^{i \infty} \frac{\Gamma(a-s) \Gamma(b-s) \Gamma(s)}{\Gamma(c-s)}(-z)^{-s} \mathrm{~d} s,
$$

Computing the residues at the simple poles of $\Gamma(s),\{0,-1,-2, \cdots\}$, we have (4) equal to (7) (a proof is given in 2.4.5) but for this case only. It can be shown, again, using (7), that $H S_{(2,1)}(a, b ; c ; z)$ can be extended to a function analytic in the complex plane, with a cut along $[1, \infty)$.

Mathai and Saxena ([23], p. 165) gave results in the case where $a$ and $b$ differ by integers and some poles become multiple. Cases $b=a+m, \quad b=a+m$ with $c=a+m+n, c=a+b+m$, etc. were considered, and gave results distinct from the series $H S_{(2,1)}(a, b ; c ; z)$. 
Example 2: For $a=3, b=a+3, c=15.3$, for example, we have $I_{1}(z)=I_{2}(z)$ on $(-\infty, 1)$, and $I_{1}(z)=I_{2}(z)=H S_{(2,1)}(z)$ on $(-1,1)$ by direct computation, and finally, $H S_{(2,1)}(z)=I_{1}(z)=I_{2}(z)$ on $(-\infty,-1)$ by analytic continuation, by taking as value of $H S_{(2,1)}(z)$ the value of $I_{1}(z)=I_{2}(z)$ within that interval. From (7) above, however, $H S_{(2,1)}(a, b ; c ; z)$ is not defined for $0<z<1$ since the formula in this case contains $\log (-z)$. This is a drawback from Mellin-Barnes representation.

Mellin-Barnes integral formula has its origins in the work of Pincherle in 1888 (see Mainardi and Pagnini [24]) and this formula was developed later by Mellin and Barnes. Athough (7) is very convenient to deal with when extensions of $H S_{(2,1)}(a, b ; c ; z)$ to forms which are more general are considered, (7) itself is seldom encountered in statistics.

\subsubsection{Contiguous Relations}

Let ${ }_{2} F_{1}(a, b ; c ; z)$ be Gauss hypergeometric function and the associated six functions, called contiguous functions: ${ }_{2} F_{1}(a \pm 1, b ; c ; z),{ }_{2} F_{1}(a, b \pm 1 ; c ; z),{ }_{2} F_{1}(a, b ; c \pm 1 ; z)$. It can be shown that ${ }_{2} F_{1}(a, b ; c ; z)$ can be obtained as a linear combination of any two of these functions, with rational coefficients expressed in terms of $a, b, c$ and $z$. There are hence 15 such relations, that can be generalized to ${ }_{2} F_{1}(a+m, b+k ; c+s ; z)$, with $m, k$ and $s$ being integers.

\subsection{Generalization to Several Parameters}

\subsubsection{Generalized Hypergeometric Functions}

Although ${ }_{q+1} F_{q}\left(a_{1}, \cdots, a_{q+1} ; b_{1}, \cdots, b_{q} ; z\right)$ is the direct generalization of Gauss ${ }_{2} F_{1}(a, b ; c ; z)$ we have, in general, the hypergeometric function in one scalar variable and $(p+q)$ parameters, defined as the series with expression:

$$
H S_{(p, q)}\left(a_{1}, \cdots, a_{p} ; b_{1}, \cdots, b_{q} ; z\right)=\sum_{n=0}^{\infty} \frac{\left(a_{1}\right)_{n} \cdots\left(a_{p}\right)_{n}}{\left(b_{1}\right)_{n} \cdots\left(b_{q}\right)_{n}} \frac{z^{n}}{n !}, \quad\left\{a_{i}\right\},\left\{b_{i}\right\} \in \mathbb{R}, z \in \mathbb{C}
$$

with Pochhammer's notation:

$$
(a)_{n}=\frac{1}{\Gamma(a)} \int_{0}^{\infty} \mathrm{e}^{-t} t^{a+n-1} d t=a(a+1) \cdots(a+n-1)=\Gamma(a+n) / \Gamma(a), \text { with }(a)_{0}=1 .
$$

$H S_{(p, q)}\left(a_{1}, \cdots, a_{p} ; b_{1}, \cdots, b_{q} ; z\right)$ converges for all $z$ when $q \geq p$, and diverges for $p>q+1$. For $p=q+1$, it is absolutely convergent for $|z|=1$ if $\operatorname{Re}(\beta)<0$, conditionally convergent for $z=-1$ if $0 \leq \operatorname{Re}(\beta)<1$ and divergent for $|z|=1$ if $1 \leq \operatorname{Re}(\beta)$. Here $\beta=\sum_{j=1}^{p} a_{j}-\sum_{j=1}^{q} b_{j}$.

For particular values of $\mathrm{p}$ and $\mathrm{q}$ we have the following series:

$$
\begin{aligned}
& { }_{0} F_{0}(. ; z)=\sum_{k=0}^{\infty} \frac{z^{k}}{k !}=\mathrm{e}^{z}, \\
& { }_{1} F_{0}(a ; z)=\sum_{k=0}^{\infty} \frac{(a)_{k} z^{k}}{k !}=(1-z)^{-a},
\end{aligned}
$$




$$
\begin{aligned}
& { }_{0} F_{1}(c ; z)=\sum_{k=0}^{\infty} \frac{z^{k}}{(c)_{k} k !}=\frac{\Gamma(c)}{z^{(c-1) / 2}} I_{(c-1)}(2 \sqrt{z}), I_{(v)}=\text { Bessel function, } \\
& { }_{1} F_{1}(a ; c ; z)=\sum_{k=0}^{\infty} \frac{(a)_{k} z^{k}}{(c)_{k} k !}=\text { Kummer } \Phi(a ; c ; z) \text { function, } \\
& { }_{2} F_{0}(a, b ;-; z)=\text { Carlson's function, } \\
& { }_{2} F_{1}(a, b ; c ; z)=\sum \frac{(a)_{k}(b)_{k}}{(c)_{k}} \frac{z^{k}}{k !}, \text { Gauss function. }
\end{aligned}
$$

\subsubsection{Analytic Continuation}

Series are very useful in the resolution of differential or algebraic equations, but to study the solution's analytic properties we rather use its integral form.

As we have seen, conditional on the values of $a, b$ and $c$ in ${ }_{2} F_{1}($.$) , integral (5) or (5')$ converges for any value of $z$, except on $[1, \infty)$ which means that the function can be extended to any point in the complex plane, with the cut $[1, \infty)$, provided $c \neq 0,-1,-2, \cdots$

For the general case, Olsson [25] proposed to express ${ }_{p} F_{q}$ as an expression of ${ }_{p-1} F_{q-1}$ progressively down to Gauss ${ }_{2} F_{1}$ (for $p=q+1$ ), using (13), the analytic continuation of which has been made. For an extensive study, and lists of properties and formulas of ${ }_{p} F_{q}$, we refer to Mathai and Saxena ([23], sect 5). As before, we have three types of integral representation:

\subsubsection{Euler Integral Representation}

$$
\begin{aligned}
& { }_{p+1} F_{q+1}\left[\begin{array}{l}
a_{1}, \cdots, a_{p}, c \\
b_{1}, \cdots, b_{q}, d
\end{array} ;\right] \\
& \left.=\frac{\Gamma(d)}{\Gamma(c) \Gamma(d-c)} \int_{0}^{1} t^{c-1}(1-t)^{d-c-1}{ }_{p} F_{q}\left[\begin{array}{l}
a_{1}, \cdots, a_{p} \\
b_{1}, \cdots, b_{q}
\end{array}\right] \mathrm{tz}\right] \mathrm{d} t, \operatorname{Re}(d)>\operatorname{Re}(c)>0
\end{aligned}
$$

\subsubsection{Laplace Representation on the Positive Axis}

a) Laplace integral representation:

$$
{ }_{p+1} F_{q}\left[\begin{array}{c}
a_{0}, a_{1}, \cdots, a_{p} \\
b_{1}, \cdots, b_{q}
\end{array}\right]=\frac{1}{\Gamma\left(a_{0}\right)} \int_{0}^{\infty} \mathrm{e}^{-t} t^{a_{0}-1} \cdot{ }_{p} F_{q}\left[\begin{array}{l}
a_{1}, \cdots, a_{p} \\
b_{1}, \cdots, b_{q}
\end{array} ; t z\right] \mathrm{d} t
$$

This relation is not to be mistaken as the Laplace transform below.

\section{b) Laplace transforms:}

Considering ${ }_{p} F_{q+1}$ and ${ }_{p+1} F_{q}$, using Laplace transforms, we have the couple:

$$
\begin{aligned}
& { }_{p+1} F_{q}\left[\begin{array}{c}
a_{1}, \cdots, a_{p}, c \\
b_{1}, \cdots, b_{q}
\end{array} ;-x^{-1}\right]=\frac{x^{c}}{\Gamma(c)} \int_{0}^{\infty} \mathrm{e}^{-t x} t^{c-1}{ }_{p} F_{q}\left[\begin{array}{l}
a_{1}, \cdots, a_{p} \\
b_{1}, \cdots, b_{q}
\end{array}-t\right] \mathrm{d} t, \\
& { }_{p} F_{q+1}\left[\begin{array}{c}
a_{1}, \cdots, a_{p} \\
b_{1}, \cdots, b_{q}, c
\end{array} ;-t\right]=\frac{\Gamma(c)}{t^{1-c} 2 \pi i} \int_{L} \mathrm{e}^{t z} z^{-c}{ }_{p} F_{q}\left[\begin{array}{l}
a_{1}, \cdots, a_{p} \\
b_{1}, \cdots, b_{q}
\end{array}-z^{-1}\right] \mathrm{d} z,
\end{aligned}
$$

where $L$ is a curve in the complex plane, properly indented to separate the two kinds of poles. 
(The above expressions become Laplace and inverse Laplace transforms of ${ }_{p} F_{q}($. when $c=1$ and $c=0$ respectively. They would permit us to "circulate" between $(p+1, q),(p, q+1)$, and $(p, q)$, under some conditions on the values of $p$ and $q$.)

\subsubsection{Mellin-Barnes Representation}

Conversely, it can be shown that if $a_{i}, b_{j} \in R$ are distinct of each other with differences different from integers, (8) is the sum of all residues of $\Gamma(s)$. Evaluating

$$
f(z)=\frac{1}{2 \pi i} \int_{d-i \infty}^{d+i \infty} \frac{\Gamma\left(a_{1}-s\right) \cdots \Gamma\left(a_{p}-s\right)}{\Gamma\left(b_{1}-s\right) \cdots \Gamma\left(b_{q}-s\right)} \Gamma(s)(-z)^{-s} \mathrm{~d} s,
$$

where $a_{i}, b_{j}$ are positive numbers, we have simple poles of $\Gamma(s)$ at $s=-\gamma, \gamma=0,1,2, \cdots$, being negative integers. Using the formula for residue value, we have:

$$
\operatorname{Res}(\gamma)=\frac{(-1)^{\gamma}}{\gamma !} \frac{\Gamma\left(a_{1}+\gamma\right) \cdots \Gamma\left(a_{p}+\lambda\right)}{\Gamma\left(b_{1}+\gamma\right) \cdots \Gamma\left(b_{q}+\gamma\right)}(-z)^{\gamma}=\left\{\frac{\prod_{j=1}^{p} \Gamma\left(a_{j}\right)}{\prod_{j=1}^{q} \Gamma\left(b_{j}\right)}\right\} \frac{\left(a_{1}\right)_{\gamma} \cdots\left(a_{p}\right)_{\gamma}}{\left(b_{1}\right)_{\gamma} \cdots\left(b_{q}\right)_{\gamma}} \frac{z^{\gamma}}{\gamma !} .
$$

Since the poles are in infinite numbers, we can see that

$$
\begin{aligned}
& \sum_{\gamma=0}^{\infty} \operatorname{Res}(\gamma)=\sum_{\gamma=0}^{\infty} \frac{\left(a_{1}\right)_{\gamma} \cdots\left(a_{p}\right)_{\gamma}}{\left(b_{1}\right)_{\gamma} \cdots\left(b_{q}\right)_{\gamma}} \frac{z^{\gamma}}{\gamma !}, \\
& { }_{p} F_{q}\left(a_{1}, \cdots, a_{p} ; b_{1}, \cdots, b_{q} ; z\right)=\frac{\prod_{j=1}^{q} \Gamma\left(b_{j}\right)}{\prod_{j=1}^{p} \Gamma\left(a_{j}\right)} \frac{1}{2 \pi i} \int_{d-i \infty}^{d+i \infty} \frac{\Gamma\left(a_{1}-s\right) \cdots \Gamma\left(a_{p}-s\right)}{\Gamma\left(b_{1}-s\right) \cdots \Gamma\left(b_{q}-s\right)} \Gamma(s)(-z)^{-s} \mathrm{~d} s
\end{aligned}
$$

NOTE: We have most common functions in mathematics represented by ${ }_{2} F_{1}(a, b ; c ; z)$ where $a, b$ and $c$ take simple values. For example, we have:

$\arctan (z)={ }_{2} F_{1}\left(1 / 2,1 ; 3 / 2 ;-z^{2}\right)$. A list of standard mathematical functions expressed as $G$-functions can be found in Mathai and Saxena ([23], sect. 2.6). Conversely, section 2.7 there gives $G$-functions expressed in terms of standard functions. Also, the software MAPLE allows us to convert a hypergeometric function into a standard function. For example, the command:

convert (hypergeom $([1,1],[2],-x)$, StandardFunctions);

gives as answer: $\frac{\ln (x+1)}{x}$.

\subsection{Generalization to G and H Functions}

In an effort to generalize ${ }_{p} F_{q}(\cdot)$ and make sense of the case $p>q+1$, we define the $H$-function, using Mellin-Barnes formula, and consider the ratio of two products of gamma functions as integrand. Fox's $H$-function, is hence defined as the integral along the complex contour $L$, of the expression $\varphi(\cdot) x^{-s}$, i.e. 


$$
H^{p} \quad \stackrel{q}{r}\left[x \mid \begin{array}{l}
\left(a_{1}, \alpha_{1}\right), \cdots,\left(a_{p}, \alpha_{p}\right) \\
\left(b_{1}, \beta_{1}\right), \cdots,\left(b_{q}, \beta_{q}\right)
\end{array}\right]=\frac{1}{2 \pi i} \int_{L} \varphi\left(\begin{array}{c}
\left(a_{1}, \alpha_{1}\right), \cdots,\left(a_{p}, \alpha_{p}\right) \\
\left(b_{1}, \beta_{1}\right), \cdots,\left(b_{q}, \beta_{q}\right)
\end{array}\right) x^{-s} \mathrm{~d} s,
$$

where

$$
\varphi\left(\begin{array}{c}
\left(a_{1}, \alpha_{1}\right), \cdots,\left(a_{p}, \alpha_{p}\right) \\
\left(b_{1}, \beta_{1}\right), \cdots,\left(b_{q}, \beta_{q}\right)
\end{array}\right)=\frac{\prod_{j=1}^{m} \Gamma\left(b_{j}+\beta_{j} s\right) \prod_{j=1}^{r} \Gamma\left(1-a_{j}-\alpha_{j} s\right)}{\prod_{j=m+1}^{q} \Gamma\left(1-b_{j}-\beta_{j} s\right) \prod_{j=r+1}^{p} \Gamma\left(a_{j}+\alpha_{j} s\right)} .
$$

The Meijer function $G(x)$ is a special case, when $\alpha_{i}=\beta_{j}=1, \forall i, j$, of $H(x)$. We notice that (15) is just one way to generalize the integrant in (13).

From (3) and (14) we can see that $G$ and $H$-functions are Inverse Mellin Transforms of $\varphi($.$) and that the Mellin-Barnes integral is now taken as the definition of the$ $G$-function, instead of a series, or a definite integral, as in preceding sections. But under some mild conditions on $a_{i}, b_{j}$, the $G^{p} q+1($.$) function can be expressed as a { }_{p} F_{q}($. function and conversely:

$$
G^{p} \quad \stackrel{p+1}{1+1}\left[-z \mid \begin{array}{c}
1-a_{1}, \cdots, 1-a_{p} \\
0,1-b_{1}, \cdots, 1-b_{q}
\end{array}\right]=\frac{\prod_{j=1}^{p} \Gamma\left(a_{j}\right)}{\prod_{j=1}^{q} \Gamma\left(b_{j}\right)} \cdot{ }_{p} F_{q}\left[\begin{array}{l}
a_{1}, \cdots, a_{p} \\
b_{1}, \cdots, b_{q}
\end{array}\right] .
$$

The $G$-function converges when $L$ is taken as one of the two paths $L_{\infty}, L_{-\infty}$ encircling the right poles (related to $a_{j}$ ), or the left poles (related to $b_{j}$ ), defining $G(z)$ for $|z|<1$ and $|z| \geq 1$ respectively, depending on the values of $p$ and $q$, or a third path $L^{*}$ can be taken as the vertical axis, separating them, for $p+q<2(m+n)$ and $|\arg (z)|<\delta \pi$, with $\delta=m+n-(p+q) / 2$, following Jordan's lemma. For discussions on the $G$-function see Mathai and Saxena [23], and on the $H$-function, see Springer [26], which also treats some uses of these functions in Statistics, as well as some computational issues. We wish to mention the following points:

1) The three paths of integration are similar to those of ${ }_{p} F_{q}(\cdot)$, and the convergence of $H$ and $G$ now depends on $\beta=\sum_{j=1}^{p} a_{j}-\sum_{j=1}^{q} b_{j}$, and also on $\lambda=\prod_{j=1}^{p} \alpha_{j}^{\alpha_{j}} / \prod_{j=1}^{q} \beta_{j}^{\beta_{j}}$.

2) There are numerous properties of the Meijer $G$-functions: Contiguity, relations with themselves, derivatives, integral transforms, etc., that we cannot list here, due to space limitation. They can be seen in Mathai and Saxena [23].

3) The $H$-function can be brought to the $G$-function for computation, when all $a_{i}, b_{j}$ are positive rational numbers, by a simple change of variable and using the multiplication formula for gamma functions.

4) The Euler and Laplace representations of $G$ involve other G-functions with lesser parameters, similarly to ${ }_{p} F_{q}().\left((9)\right.$ and $\left.\left(9^{\prime}\right)\right)$ :

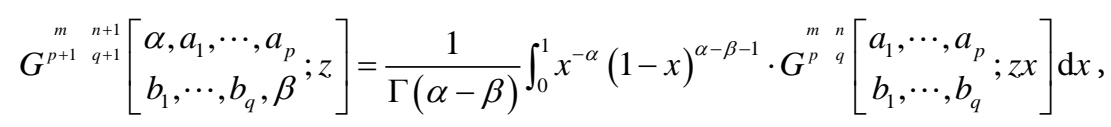




$$
\begin{aligned}
& G^{p+1} \underset{q}{n+1}\left[\begin{array}{c}
\alpha, a_{1}, \cdots, a_{p} \\
b_{1}, \cdots, b_{q}
\end{array} ; x^{-1}\right]=\int_{0}^{\infty} \mathrm{e}^{-x} y^{-\alpha} \cdot G^{m}{ }^{p} q\left[\begin{array}{l}
a_{1}, \cdots, a_{p} \\
b_{1}, \cdots, b_{q}
\end{array} ; x y\right] \mathrm{d} y, \operatorname{Re}(x)>0 . \\
& G^{m+2} \underset{q+2}{q}\left[\begin{array}{c}
0,1 / 2, a_{1}, \cdots, a_{p} \\
b_{1}, \cdots, b_{q}
\end{array} 4 \eta / z^{2}\right]=\int_{0}^{\infty} \mathrm{e}^{-z x} \cdot G^{m}{ }^{m}{ }^{n}\left[\begin{array}{l}
a_{1}, \cdots, a_{p} \\
b_{1}, \cdots, b_{q}
\end{array} ; x^{2}\right] \mathrm{d} x, \operatorname{Re}(z)>0
\end{aligned}
$$

The Laplace transforms pair of $\mathrm{G}$ :

$$
\int_{0}^{\infty} \mathrm{e}^{-x y} y^{-\alpha} \cdot G^{m} q^{m}\left[\begin{array}{l}
a_{1}, \cdots, a_{p} \\
b_{1}, \cdots, b_{q}
\end{array} ; \eta y\right] \mathrm{d} y=x^{\alpha-1} G^{m+1} \underset{q+1}{q}\left[\begin{array}{c}
\alpha, a_{1}, \cdots, a_{p} \\
b_{1}, \cdots, b_{q}
\end{array} ; \eta / x\right], \operatorname{Re}(x)>0,
$$

and its inverse

$$
x^{-\alpha} G^{p} \quad{ }_{q+1}^{n}\left[\begin{array}{c}
a_{1}, \cdots, a_{p} \\
b_{1}, \cdots, b_{q}, \alpha^{\prime}
\end{array} ; \eta x\right]=\frac{1}{2 \pi i} \int_{c-i \infty}^{c+i \infty} \mathrm{e}^{w x} w^{\alpha-1} \cdot G^{p}{\underset{q}{m}}_{m}^{n}\left[\begin{array}{l}
a_{1}, \cdots, a_{p} \\
b_{1}, \cdots, b_{q}
\end{array} ; \eta / w\right] \mathrm{d} w, c>0 .
$$

(Taking $\alpha=0$ we have the Laplace transform of $G^{p q}($.$) ).$

Also, the relation $G^{m} q_{q}^{n}\left[\begin{array}{l}a_{1}, \cdots, a_{p} \\ b_{1}, \cdots, b_{q}\end{array} ; z\right]=G^{q} \frac{n m}{p}\left[\begin{array}{l}1-b_{1}, \cdots, 1-b_{q} \\ 1-a_{1}, \cdots, 1-a_{p}\end{array} ; 1 / z\right]$ permits the analytic continuation of $G($.) from inside the unit disk $|z| \leq 1$ to outside it, with an appropriate cut, if necessary, depending on the value of $m+n-p$.

Generalizations of $H$-functions: We will not go beyond the $H$-function, but it is worth mentioning that generalized forms of $H$ exist, e.g. the one in Rathie [27], which depends on an additional set of parameters $\{\gamma, \kappa, \lambda, \zeta\}$. It is defined by:

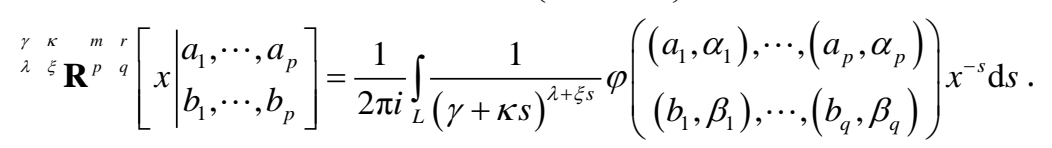

This function should not be confused with Carlson's $R_{n}(\cdot)$ function [10] defined in section 6.3.

But the Fox-Wright function

$$
{ }_{p} \psi_{q}\left[\begin{array}{l}
\left(a_{1}, A_{1}\right)\left(a_{2}, A_{2}\right) \cdots\left(a_{p}, A_{p}\right) \\
\left(b_{1}, B_{1}\right)\left(b_{2}, B_{2}\right) \cdots\left(b_{q}, B_{q}\right)
\end{array} ; z\right]=\sum_{j=0}^{\infty} \frac{\Gamma\left(a_{1}+A_{1} j\right) \cdots \Gamma\left(a_{p}+A_{p} j\right)}{\Gamma\left(b_{1}+B_{1} j\right) \cdots \Gamma\left(b_{q}+B_{q} j\right)} \frac{z^{j}}{j !}
$$

can be expressed as a H-function, while the MacRobert E-function, defined below, can be expressed as a G-function.

$$
\begin{aligned}
E\left[\begin{array}{l}
a_{1}, \cdots, a_{p} \\
b_{1}, \cdots, b_{q}
\end{array}\right] x & \\
= & \frac{\prod_{i=1}^{p} \Gamma\left(a_{i}\right)}{\prod_{i=1}^{q} \Gamma\left(b_{i}\right)} \cdot{ }_{p} F_{q}\left(\begin{array}{l}
a_{1}, \cdots, a_{p} \\
b_{1}, \cdots, b_{q}
\end{array} \mid-x^{-1}\right), p \leq q, x \neq 0 \text {, or } p=q+1,|x|>1 \\
= & \sum_{h=1}^{p} \frac{\prod_{i=1}^{p} \Gamma\left(a_{i}-a_{h}\right)^{*} \Gamma\left(b_{i}-a_{h}\right)}{q} \cdot \Gamma\left(a_{h}\right) x^{a_{h}}{ }_{q+1} F_{p-1}\left(\begin{array}{c}
a_{h}, 1+a_{h}-b_{1}, \cdots, 1+a_{h}-b_{q} \\
1+a_{h}-a_{1}, \cdots, *, \cdots, 1+a_{h}-a_{p}
\end{array} \mid(-1)^{p-q} x\right), \\
& *=\text { ignor } j=h \text { contrib }, p \geq q+2 \text { or } p \geq q+1 \&|x|<1 .
\end{aligned}
$$




\section{Hypergeometric Series and Functions in Several Independent Scalar Variables}

When we go from one variable to two variables there are different ways to sum the variables, reflected in different expressions for the coefficients given to $\frac{x^{n}}{n !} \frac{y^{m}}{m !}$, and hence, we have different functions. In two variables, we have Appell hypergeometric functions, defined as follows:

\subsection{Appell, Lauricella and Other Sums}

$$
\begin{aligned}
& F_{1}\left(a, b, b^{\prime} ; c ; x, y\right)=\sum_{m, n=0}^{\infty} \frac{(a)_{m+n}(b)_{m}\left(b^{\prime}\right)_{n}}{(c)_{m+n}} \frac{x^{n}}{n !} \frac{y^{m}}{m !}, \max (|x|,|y|)<1, \\
& F_{2}\left(a, b, b^{\prime} ; c, c^{\prime} ; x, y\right)=\sum_{m, n=0}^{\infty} \frac{(a)_{m+n}(b)_{m}\left(b^{\prime}\right)_{n}}{(c)_{m}\left(c^{\prime}\right)_{n}} \frac{x^{n}}{n !} \frac{y^{m}}{m !},|x|+|y|<1, \\
& F_{3}\left(a, a^{\prime} ; b, b^{\prime} ; c ; x, y\right)=\sum_{m, n=0}^{\infty} \frac{(a)_{m}\left(a^{\prime}\right)_{n}(b)_{m}\left(b^{\prime}\right)_{n}}{(c)_{m+n}} \frac{x^{n}}{n !} \frac{y^{m}}{m !}, \max (|x|,|y|)<1, \\
& F_{4}\left(a, b ; c, c^{\prime} ; x, y\right)=\sum_{m, n=0}^{\infty} \frac{(a)_{m+n}(b)_{m+n}}{(c)_{m}\left(c^{\prime}\right)_{n}} \frac{x^{n}}{n !} \frac{y^{m}}{m !}, \sqrt{|x|}+\sqrt{|y|}<1 .
\end{aligned}
$$

a) Each of these functions can be expressed as an infinite series in $x$ alone, with coefficients containing Gauss function ${ }_{2} F_{1}(\cdot, y)$. For example, we have:

$$
F_{1}\left(a, b, b^{\prime} ; c ; x, y\right)=\sum_{m=0}^{\infty} \frac{(a)_{m}(b)_{m}}{(c)_{m}} \cdot{ }_{2} F_{1}\left(a+m, b^{\prime} ; c+m ; y\right) \frac{x^{m}}{m !},
$$

and, similarly for other functions.

Also, $F_{1}(\cdot)$ and its generalization $F_{D}(\cdot)$ (see sect. 3.2), seem to be the most important among these functions, with numerous applications in several disciplines.

b) Other hypergeometric functions, 34 in total, have been defined by Jacob Horn. The main ones are $G_{1}(\cdot), G_{2}(\cdot), G_{3}(\cdot)$ and $H_{1}(\cdot), H_{2}(\cdot), \cdots, H_{7}(\cdot)$. They will not be treated here. Whittaker, Pandey, Srivastava, Wright, Macrobert, Kampé de Fériet, and Lauricella-Saran functions, as well as lesser-known functions, will not be treated either, due to space limitation, see Exton [28].

c) Functions $\Psi(\cdot)$ and $\Phi(\cdot)$ of Humbert: These 7 confluent forms of the Appell series are denoted $\Phi_{1}, \Phi_{2}, \Phi_{3}, \Psi_{1}, \Psi_{2}, \Xi_{1}, \Xi_{2}$, and are limiting values of Appell functions. For example:

$$
\Phi_{2}\left[\beta_{1}, \beta_{2} ; \gamma ; x, y\right]=\sum_{m, n=0}^{\infty} \frac{\left(\beta_{1}\right)_{m}\left(\beta_{2}\right)_{n}}{(\gamma)_{m+n}} \frac{x^{n}}{n !} \frac{y^{m}}{m !}=\lim _{|\alpha| \rightarrow \infty} F_{1}\left[\alpha, \beta_{1}, \beta_{2} ; \gamma ; x / \alpha, y / \alpha\right] .
$$

They have a particular role in the representation of Appell functions. For example, we have $F_{1}(\cdot)$ as a function of $\Phi_{2}(\cdot)$. The corresponding 13 confluent forms of the Horn series, denoted $\Gamma_{1}, H_{1}, \cdots, H_{11}, \Phi_{2}$, will not be discussed in detail here. We refer to Srivastava and Karlsson [29] for these functions. 


\subsection{Integral Representations and Further Generalization}

Lauricella functions are extensions of Appell functions to $n$ variables, where $n>2$, with $F_{A}\left(a, b_{1}, \cdots, b_{n} ; c_{1}, \cdots, c_{n} ; x_{1}, \cdots, x_{n}\right), F_{B}\left(a_{1}, \cdots, a_{n} ; b_{1}, \cdots, b_{n} ; c ; x_{1}, \cdots, x_{n}\right)$, $F_{C}\left(a, b ; c_{1}, \cdots, c_{n} ; x_{1}, \cdots, x_{n}\right)$ and $F_{D}\left(a, b_{1}, \cdots, b_{n}, c ; x_{1}, \cdots, x_{n}\right)$ corresponding, respectively, to Appell functions, $F_{2}\left(a, b_{1}, b_{2} ; c_{1}, c_{2} ; x_{1}, x_{2}\right), F_{3}\left(a_{1}, a_{2} ; b_{1}, b_{2} ; c ; x_{1}, x_{2}\right)$, $F_{4}\left(a, b ; c_{1}, c_{2} ; x_{1}, x_{2}\right)$ and $F_{1}\left(a, b_{1}, b_{2} ; c ; x_{1}, x_{2}\right)$ in 2 variables.

And the Humbert function in $n$ variables is defined as follows:

$$
\Phi_{2}^{(n)}\left(b_{1}, \cdots, b_{n} ; c ; x_{1}, \cdots, x_{n}\right)=\sum_{m, n=0}^{\infty} \frac{\left(b_{1}\right)_{m_{1}} \cdots\left(b_{n}\right)_{m_{n}}}{(c)_{m_{1}+\cdots+m_{n}}} \frac{x_{1}^{m_{1}} \cdots x_{n}^{m_{n}}}{m_{1} ! \cdots m_{n} !},
$$

\subsubsection{Integral Representation of Euler Type}

These integrals represent hypergeometric functions in $n$ variables. For example,

$$
\begin{aligned}
& F_{D}\left(a, b_{1}, \cdots, b_{n} ; c ; x_{1}, \cdots, x_{n}\right) \\
& =\frac{\Gamma(c)}{\Gamma\left(c-b_{1}-\cdots-b_{n}\right) \prod_{i=1}^{n} \Gamma\left(b_{i}\right)} \int_{0}^{1} \cdots \int_{0}^{1} \prod_{j=1}^{n} u_{j}^{b_{j}-1}\left(1-u_{1}-\cdots-u_{n}\right)^{c-b_{1}-\cdots-b_{n}-1}\left(1-u x_{1}-\cdots-u x_{n}\right)^{-a} \mathrm{~d} u_{1} \cdots \mathrm{d} u_{n},
\end{aligned}
$$

and similarly for other functions, which can serve to extend the function outside the domains of convergence of the series. The $n$-tuple $\left(S_{1}, \cdots, S_{n}\right)$, where $S_{i}$ is either 0,1 or $\infty$, are the regular singularities for the analytic extensions, and should be studied separately (see Exton ([28], sect 6.7.4) for the case $n=3$ and $F_{D}($.$) ).$

In particular, for $F_{D}($.$) , or F_{1}($.$) , it can be represented by a single integral, a result$ known as Picard's Theorem 9 (although the result seemed to have been established eight years earlier). We have:

$$
F_{D}\left(a, b_{1}, \cdots, b_{n} ; c ; x_{1}, \cdots, x_{n}\right)=\frac{\Gamma(c)}{\Gamma(a) \Gamma(c-a)} \int_{0}^{1} u^{a-1}(1-u)^{c-a-1}\left(1-u x_{1}\right)^{-b_{1}} \cdots\left(1-u x_{n}\right)^{-b_{n}} \mathrm{~d} u \text {. }
$$

But deeper results are obtained using A-hypergeometric functions (see section 8.1.2). Also, $F_{1}(\cdot)$ has strong connections with elliptic integrals. For example, we have:

$$
\int_{0}^{\pi / 2} \frac{\mathrm{d} \theta}{\left(1-n \sin ^{2} \theta\right) \sqrt{1-(k \sin \theta)^{2}}}=\frac{\pi}{2} F_{1}\left(\frac{1}{2}, 1, \frac{1}{2} ; n, k^{2}\right) .
$$

Convenient forms for these integrals have been suggested by Carlson, using his own hypergeometric functions (see sect. 6.3.3).

\subsubsection{Integral Representation of Laplace Type on $(0, \infty)^{n}$}

Lauricella functions are expressed in terms of $n$-fold integrals of $\Psi_{2}, \Phi_{2},{ }_{0} F_{1}$ and ${ }_{1} F_{1}$, respectively.

Again, for $F_{D}$ we have a multiple integral expression:

$$
F_{D}\left(a, b_{1}, \cdots, b_{n} ; c ; x_{1}, \cdots, x_{n}\right)=\frac{1}{\prod_{i=1}^{n} \Gamma\left(b_{i}\right)} \int_{0}^{\infty} \cdots \int_{0}^{\infty} \mathrm{e}^{-t_{1}-\cdots-t_{n}} \prod_{j=1}^{n} t_{j}^{b_{j}-1} \cdot{ }_{1} F_{1}\left(a ; c ; x_{1} t_{1}+\cdots+x_{n} t_{n}\right) \mathrm{dt} t_{1} \cdots \mathrm{d} t_{n}
$$


and also a single integral representation, using Humbert function:

$$
F_{D}\left(a, b_{1}, \cdots, b_{n} ; c ; x_{1}, \cdots, x_{n}\right)=\frac{1}{\Gamma(a)} \int_{0}^{\infty} \mathrm{e}^{-t} t^{a-1} \Phi_{2}^{(n)}\left(b_{1}, \cdots, b_{n} ; c ; x_{1} t, \cdots, x_{n} t\right) \mathrm{d} t .
$$

\subsubsection{Representation of Mellin-Barnes Type}

Integrals are taken along the infinite imaginary axis, suitably indented. For example, for

$F_{D}(\cdot)$ we have

$$
\begin{aligned}
& F_{D}\left(a, b_{1}, \cdots, b_{n} ; c ; x_{1}, \cdots, x_{n}\right) \\
& =\frac{\Gamma(c)}{\Gamma(a) \prod_{j=1}^{n} \Gamma\left(b_{j}\right)} \frac{1}{(2 \pi i)^{n}} \int_{\mathrm{I}_{m}} \cdots \int_{\mathrm{I}_{m}} \frac{\Gamma\left(a-\left(t_{1}+\cdots+t_{n}\right)\right) \prod_{j=1}^{n} \Gamma\left(b_{j}-t_{j}\right)}{\Gamma\left(c-\left(t_{1}+\cdots+t_{n}\right)\right)} \prod_{j=1}^{n} \Gamma\left(t_{j}\right) \prod_{j=1}^{n}\left(-x_{j}\right)^{-t_{j}} \mathrm{~d} t_{1} \cdots \mathrm{d} t_{n} .
\end{aligned}
$$

Analytic continuation for Appell and Lauricella series: They can be continued analytically outside their convergence domain using their Euler integral representation or recurrence relations that exist between themselves. Exton ([28], sect 6.6) discusses this topic in details. In particular, the case of $F_{D}^{(3)}(\cdot)$ is carefully presented.

The presence of so many forms of hypergeometric functions in $n$ variables is embarrassing when we do not know the relations between them, which was the situation in the first half of the 20th century. But this situation started to change by the mid-eighties (see sect. 8.1.3).

\subsection{Differential Equations and Systems}

Partial and ordinary differential equations play an important role in Applied mathematics and to a lesser extent, in Statistics. They still constitute a major tool in the study of hypergeometric functions in pure and applied mathematics.

a) The basic hypergeometric equation (of Fuchsian type) in one variable is:

$$
x(1-x) y^{\prime \prime}+[c-(a+b+1) x] y^{\prime}-a b y=0,
$$

a solution of which, obtained under series form, is $y_{1}=H S_{(2,1)}(a, b ; c ; x)$. Every second-order linear ODE with three regular singular points can be transformed into this equation. There is an extensive discussion in the literature (e.g. Lebedev [19]) on values of this solution at regular singularities 0,1 and $\infty$, as well as when there are relations between coefficients containing integers. When $c$ is not an integer the other solution independent of the first is: $y_{2}=x^{1-c}{ }_{2} F_{1}(1-c+a, 1-c+b ; 2-c ; x)$. The general solution of (21) is hence: $y=c_{1} y_{1}+c_{2} y_{2}$, with $c_{1}, c_{2}$ constants.

Concerning other hypergeometric functions, the equation satisfied by $G$-functions is:

$$
\left[(-1)^{m+n-p} z \prod_{j=1}^{p}\left(z \frac{\mathrm{d}}{\mathrm{d} z}-a_{j}+1\right)-\prod_{k=1}^{q}\left(z \frac{\mathrm{d}}{\mathrm{d} z}-b_{k}\right)\right] u(z)=0,
$$

and, for partial differential systems, there is one for each Lauricella function $F_{A}, F_{B}, F_{C}$ and $F_{D}$. For this last function $F_{D}$, it is, for example:

$x_{j}\left(1-x_{j}\right) \frac{\partial^{2} F}{\partial x_{j}^{2}}+\left(1-x_{j}\right) \sum_{k=1, k \neq j}^{n} x_{k} \frac{\partial^{2} F}{\partial x_{k} \partial x_{j}}+\left[c-\left(a+1+b_{j}\right) x_{j}\right] \frac{\partial F}{\partial x_{j}}-b_{j} \sum_{k=1, k \neq j}^{n} x_{k} \frac{\partial F}{\partial x_{k}}-a b_{j} F=0$. 
The resolution of these systems is not simple and there are up to sixty solutions. Basically, there are several independent solutions which include the hypergeometric series obtained when using infinite series in searching for solutions. We invite the reader to consult Exton ([28], Chapter 5). We will again mention differential equations since these pde's will be at the heart of A-hypergeometric systems presented later.

b) The differential equation satisfied by ${ }_{p+1} F_{p}($.$) is$

$$
\left\{\vartheta\left(\vartheta+a_{1}-1\right) \cdots\left(\vartheta+a_{p}-1\right)-z\left(\vartheta+b_{1}\right) \cdots\left(\vartheta+b_{p+1}\right)\right\} y=0,
$$

where $\vartheta=z \frac{\mathrm{d}}{\mathrm{d} z}$. There are $p$ more solutions if all $a_{i}$ are not integers. They are independent, when the difference between any two of the values: $1, a_{1}, \cdots, a_{p}$ is not an integer.

Differential equations for one-matrix hypergeometric functions can be considered. A short introduction to this topic is given by Muirhead ([30], chapter 7). Also

\subsection{Generalized $\mathrm{G}$ and $\mathrm{H}$ functions in Several Independent Scalar Variable}

As for one variable, we use the Mellin-Barnes approach to define this function. Buschman [31] defined $H$-functions of 2 variables as an integral in the complex planes of a ratio $\theta(s, t)$ of two products, i.e.

$$
H\left[(x, y) \mid \begin{array}{c}
\left(\alpha_{1}, a_{1}, A_{1}\right), \cdots,\left(\alpha_{m}, a_{m}, A_{m}\right) \\
\left(\beta_{1}, b_{1}, B_{1}\right), \cdots,\left(\beta_{n}, b_{n}, B_{n}\right)
\end{array}\right]=\frac{1}{(2 \pi i)^{2}} \int_{L_{S}} \int_{L_{T}} \theta(s, t) x^{-s} y^{-t} \mathrm{~d} s \mathrm{~d} t,
$$

where $L_{S}, L_{T}$ are curves in the two complex planes, and

$$
\theta(s, t)=\frac{\prod_{j=1}^{m} \Gamma\left(\alpha_{j}+a_{j} s+A_{j} t\right)}{\prod_{j=1}^{n} \Gamma\left(\beta_{j}+b_{j} s+B_{j} t\right)} .
$$

But, as pointed out by Nguyen Thanh Hai and Yakubovich [32], the representation as the residue sum still has difficulties. There are some results on the Cauchy integral formula for several complex variables but it is still unclear how the residues can be computed in the general case. Hence, like the univariate case, not all of these integrals can be expressed as double series. Euler and Laplace representations, in function of other $H(x, y)$ functions, are quite complicated and are not given here. More information on $H(x, y)$ can be obtained from Mathai and Saxena [33]. More advanced results on $\mathrm{H}$ are presented in [34]. We will not elaborate on these results, and neither on other definitions of $H(x, y)$ encountered in the literature.

\section{Hypergeometric Functions in Matrix Arguments: Three Proposed Approaches}

In multivariate Analysis variables encountered can be matrices, which will be arguments of hypergeometric functions. 


\subsection{Functions in One Matrix Variate}

In going from a scalar variable to a matrix, there are several difficulties to define the hypergeometric function. First, functions of matrices, square or rectangular, can only be defined under certain conditions (Higham [35]), and they can be scalar-valued, or matrix-valued. Secondly, for scalar-valued matrix functions, they are usually based on symmetric functions of the matrix entries, or of the eigenvalues of the input square matrices. A simple introduction to this topic is given by Pham-Gia and Turkkan [36]. We recall here some basic notions of calculus on matrices, that are not so obvious.

Domain of integration: Let $f(X)$ be a scalar function of the matrix $X$. Then $\int_{\Omega} f(X) \mathrm{d} X$ is the iterated integral of $f(X)$ for each entry of $X$ separately, over the region $\Omega$ located within the space defined by the simplex bounding the ranges of the entries of $X$.

Since it is usually very difficult to carry out direct integration over a complex region $\Omega$, integration on simple regions are frequently done by changes of variables, matrix decompositions, and finally identification with known expressions.

We have also the region $0<X<I_{m}$ as the set of all square matrices such that $X$ and $I_{m}-X$ are positive definite, which reduces to the continuous variable $\mathrm{x}$ being between 0 and 1 in a unidimensional space.

Jacobian and Exterior product: In carrying out the required changes of variables mentioned above we have to use jacobians, and using wedge products $\mathrm{d} x_{1} \wedge \mathrm{d} x_{2}=-\mathrm{d} x_{2} \wedge \mathrm{d} x_{1}$ and exterior forms would be helpful. We have, for example, for $I=\int_{A} f\left(x_{1}, \cdots, x_{m}\right) \mathrm{d} x_{1} \wedge \cdots \wedge \mathrm{d} x_{m}$ and transforms $x_{i}=x_{i}\left(y_{1}, \cdots, y_{m}\right), i=1, \cdots, m$, the result $I=\int_{A} g\left(y_{1}, \cdots, y_{m}\right) \mathrm{d} y_{1} \wedge \cdots \wedge \mathrm{d} y_{m}$, with $\underset{i=1}{m} \mathrm{~d} x_{i}=\operatorname{det}\left(\frac{\partial x_{i}}{\partial y_{j}}\right) \underset{i=1}{\wedge} \mathrm{d} y_{i}$ where the jacobian of the transformation is the absolute value of the determinant $\operatorname{det}\left(\frac{\partial x_{i}}{\partial y_{j}}\right)$.

The multigamma function: Let $f(X)=e \operatorname{tr}(-X)|X|^{a-\frac{m+1}{2}}$, where etr $(X)$ is the exponential of the trace of $X$, with the domain of positive definite matrices being $\Omega=\{X: X>0\}$, we have the multivariate gamma function $\Gamma_{m}(a)=\int_{X>0} \operatorname{etr}(-X)|X|^{a-(m+1) / 2} \mathrm{~d} X$. Carrying out integration as explained above, we obtain a product of $m$ ordinary gamma functions $\Gamma_{m}(a)=\pi^{m(m-1) / 2} \prod_{i=1}^{m} \Gamma\left(a-\frac{i-1}{2}\right)$.

The Matrix Laplace Transform: Let $f(S)$ be a scalar function of the positive definite symmetric $m \times m$ matrix $S$. Its Laplace transform is defined by $g(Z)=\int_{S>0} \operatorname{etr}(-Z S) f(S) \mathrm{d} S, Z=X+i Y$ symmetric.

We assume that the integral converges in the half-plane $\operatorname{Re}(Z)=X>X_{0}$, for some positive definite matrix $X_{0}$. Then $g(Z)$ is analytic in $Z$ in the half-plane. If $\int|g(X+i Y)| \mathrm{d} y<\infty$ and $\lim _{X \rightarrow \infty} \int|g(X+i Y)| \mathrm{d} Y=0$, then the inverse Laplace transform 
is: $\quad f(S)=\frac{1}{(2 \pi i)^{\frac{m(m+1)}{2}}} \int_{\operatorname{Re}(Z)>X_{0}>0} \operatorname{etr}(X Z) g(Z) \mathrm{d} Z$.

Gupta and Nagar [37] can be consulted for several notions on matrix variate distributions.

To define hypergeometric functions in one matrix argument, there are three approaches offered in the literature.

\subsubsection{Laplace Transform Approach}

This approach was pioneered by Bochner, developed by Herz [38], and uses the matrix forms of (10) and (11). We can then define ${ }_{p} F_{q+1}(\Lambda)$ and ${ }_{p} F_{q+1}(\Lambda)$. More precisely, we define ${ }_{p} F_{q}\left[\begin{array}{l}a_{1}, \cdots, a_{p} \\ b_{1}, \cdots, b_{q}\end{array} ; \Lambda\right]$ in a progressive way, with

$$
\begin{aligned}
& { }_{p+1} F_{q}\left[\begin{array}{c}
a_{1}, \cdots, a_{p}, \gamma \\
b_{1}, \cdots, b_{q}
\end{array} ;-Z^{-1}\right] \\
& =\operatorname{det}(Z)^{\gamma} \frac{1}{\Gamma_{m}(\gamma)} \int_{\Lambda>0} \operatorname{etr}(-\Lambda Z) \cdot{ }_{p} F_{q}\left[\begin{array}{l}
a_{1}, \cdots, a_{p} \\
b_{1}, \cdots, b_{q}
\end{array}-\Lambda\right] \operatorname{det}(\Lambda)^{\gamma-(m+1) / 2} \mathrm{~d} \Lambda \\
& { }_{p} F_{q+1}\left[\begin{array}{c}
a_{1}, \cdots, a_{p} \\
b_{1}, \cdots, b_{q}, \gamma
\end{array}-\Lambda\right] \\
& =\operatorname{det}(\Lambda)^{(m+1) / 2-\gamma} \frac{\Gamma_{m}(\gamma)}{(2 \pi i)^{m(m+1) / 2}} \int_{\operatorname{Re} Z=X_{0}>0} \operatorname{etr}(\Lambda Z)_{p} F_{q}\left[\begin{array}{l}
a_{1}, \cdots, a_{p} \\
b_{1}, \cdots, b_{q}
\end{array}-Z^{-1}\right] \operatorname{det}(Z)^{-\gamma} \mathrm{d} Z
\end{aligned}
$$

Here, $m$ is the dimension of the matrices and in (25). Also, for the multivariate Laplace transform, the elements off-diagonal of $Z$ are taken as $Z_{i j} / 2$. So, theoretically, hypergeometric functions can be defined in this way, and sometimes they can be computed by numerical methods.

\subsubsection{Zonal Polynomials Approach}

This approach was introduced by James, and developed by James and Constantine, using results on group decomposition by Lo Keng Hua (see Gross and Richards [39]). It is based on group representation using matrices, aimed at replacing $x^{n}$ of the scalar case, by a polynomial $C(X)$, when $x$ is replaced by the random matrix $X . C(X)$ is called the zonal polynomial of $X$. We have, for example, instead of the multinomial form

$$
[\operatorname{tr}(X)]^{k}=\left(x_{1}+\cdots+x_{m}\right)^{k}=\sum_{\Sigma k_{i}=k} \frac{m !}{k_{1} ! \cdots k_{m} !} x_{1}^{k_{1}} \cdots x_{m}^{k_{m}},
$$

the expression $[\operatorname{tr}(X)]^{k}=\sum_{\kappa} C_{\kappa}(X)$, where the zonal polynomial $C_{\kappa}(X)$ is a symmetric homogeneous polynomial of degree $k$ in elements of $X$. Here, $\kappa$ is the partition $\left(k_{1}, \cdots, k_{m}\right)$, with $k_{1} \geq k_{2} \geq \cdots \geq k_{m} \geq 0$, and $\sum_{i=1}^{m} k_{i}=k . V_{k}$ is the vector space of homogeneous polynomials of degree $k$ in the $m(m+1) / 2$ elements of the symmetric $m \times m$ matrix $X$, and $V_{k}=\underset{\kappa}{\oplus} V_{\kappa}$, i.e. $V_{\kappa}$ is the direct sum of irreducible invariant 
subspaces $V_{\kappa}$ in the representation of the real linear group $G l(m, \mathbb{R})$ in the vector space $V_{\kappa}$.

When $m=1$ we have indeed $x^{k}=C_{\kappa}(x)$ and hence, zonal polynomials of a matrix are similar to powers of a scalar variable.

The decomposition into a direct sum of subrings is assured by ring theory (Gross and Richards [39]) and hence, zonal polynomials do exist. However, their values must be obtained by solving a differential equation of Laplace-Beltrami type

$$
\Delta_{Y} C_{\kappa}(Y)=\alpha_{\kappa} C_{\kappa}(Y), \Delta_{Y}=\sum_{i=1}^{m} y_{i}^{2} \frac{\partial^{2}}{\partial y_{i}^{2}}+\sum_{i=1}^{m} \sum_{j=1, j \neq i}^{m} \frac{y_{i}^{2}}{y_{i}-y_{j}} \frac{\partial}{\partial y_{i}}
$$

(Muirhead [30]), which quickly becomes difficult to track. More precisely, we have:

$$
\Delta_{Y} C_{\kappa}(Y)=\left[\rho_{\kappa}+k(m-1)\right] C_{\kappa}(Y), \text { with } \rho_{\kappa}=\sum_{i=1}^{m} k_{i}\left(k_{i}-i\right) .
$$

Alternately, we can obtain $C_{\kappa}(Y)=\sum_{\lambda \leq \kappa} c_{\kappa, \lambda} M_{\lambda}(Y)$, where the monomial symmetric functions are $M_{\lambda}(Y)=\sum y_{i_{1}}^{k_{1}} \cdots y_{i_{p}}^{k_{p}}$ and the coefficients

$$
\begin{gathered}
c_{\kappa, \lambda}=\sum_{\lambda<\mu \leq \kappa} \frac{\left[\left(l_{i}+t\right)-\left(l_{j}-t\right)\right]}{\rho_{\kappa}-\rho_{\lambda}} c_{\kappa, \mu}, \lambda=\left(l_{1}, \cdots, l_{m}\right), \\
\mu=\left(l_{1}, \cdots, l_{i}+t, \cdots, l_{j}-t, \cdots, l_{m}\right), \quad t=\left(1,2, \cdots, l_{j}\right) .
\end{gathered}
$$

For $k=3$, for example, we have the values of $c_{\kappa, \lambda}$ as follows:

$$
\begin{array}{ccccc} 
& & \multicolumn{3}{c}{\lambda} \\
& & (3) & (2,1) & (1,1,1) \\
& (3) & 1 & 3 / 5 & 2 / 5 \\
& (2,1) & 0 & 12 / 5 & 18 / 5 \\
& (1,1,1) & 0 & 0 & 2
\end{array}
$$

Other methods, not necessarily simpler, have been suggested (Kates [40], Saw [41], Takemura [42]). Values of $C_{\kappa}(Y)$ up to $k=20$ are found by researchers. We have some basic results on integration associated with zonal polynomials, as follows (Muirhead [30]):

$$
\begin{gathered}
\int_{O(m)} C_{\kappa}\left(X H Y H^{\prime}\right) \mathrm{d} H=\frac{C_{\kappa}(X) C_{\kappa}(Y)}{C_{\kappa}\left(I_{m}\right)}, \\
\int_{X>0} e t r(-X Z)|X|^{a-\frac{m+1}{2}} C_{\kappa}(X Y) \mathrm{d} X=(a)_{\kappa} \Gamma_{m}(a) C_{\kappa}\left(Y Z^{-1}\right) /|Z|^{a},
\end{gathered}
$$

and

$$
\int_{0<X<I_{m}}|X|^{a-\frac{m+1}{2}}\left|I_{m}-X\right|^{b-\frac{m+1}{2}} C_{\kappa}(X Y) \mathrm{d} X=\frac{(a)_{\kappa}}{(a+b)_{\kappa}} \frac{\Gamma_{m}(a) \Gamma_{m}(b)}{\Gamma_{m}(a+b)} C_{\kappa}(Y)
$$

with

$$
\Gamma_{m}(a)=\pi^{m(m-1) / 4} \prod_{j=1}^{m} \Gamma\left(a-\frac{j-1}{2}\right) \text { and }(\alpha)_{\kappa}=\prod_{j=1}^{m}\left(\alpha-\frac{j-1}{2}\right)_{k_{j}}
$$


A hypergeometric functions of one matrix $X$ then have the familiar form:

$$
\begin{aligned}
& { }_{p} F_{q}\left(a_{1}, \cdots, a_{p} ; b_{1}, \cdots, b_{q} ; Z\right) \\
& =\sum_{k=0}^{\infty} \sum_{\kappa} \frac{\left(a_{1}\right)_{\kappa} \cdots\left(a_{p}\right)_{\kappa}}{\left(b_{1}\right)_{\kappa} \cdots\left(b_{q}\right)_{\kappa}} \frac{C_{\kappa}(Z)}{k !}
\end{aligned}
$$

and we have

$$
{ }_{2} F_{1}(a ; b ; Z)=\sum_{k=0}^{\infty} \sum_{\kappa} \frac{(a)_{\kappa}(b)_{\kappa}}{(c)_{\kappa}} \frac{C_{\kappa}(Z)}{k !} .
$$

Like the scalar variable case (see (9)), using zonal polynomials, we have the Euler-type representation:

$$
\begin{aligned}
& { }_{p+1} F_{q+1}\left[\begin{array}{l}
a_{1}, \cdots, a_{p}, c \\
b_{1}, \cdots, b_{q}, d
\end{array} ; \Lambda\right] \\
& =\frac{\Gamma_{m}(d)}{\Gamma_{m}(c) \Gamma_{m}(d-c)} \int_{0<T<I_{m}}|T|^{c-\frac{m+1}{2}}\left|I_{m}-T\right|^{d-c-\frac{m+1}{2}}{ }_{p} F_{q}\left[\begin{array}{l}
a_{1}, \cdots, a_{p} \\
b_{1}, \cdots, b_{q}
\end{array} ; \Lambda\right] \mathrm{d} T
\end{aligned}
$$

Similarly, again using zonal polynomials, the Laplace and inverse Laplace representations of ${ }_{p} F_{q+1}$ in the scalar variable case can be extended to the matrix case, and we can prove (25) and (26).

This zonal polynomials approach is favored when we aim at deriving theoretical results, using and obtaining expressions similar to the scalar case. Since higher order zonal polynomials are difficult to obtain we have here a topic still under development. It is worth mentioning that numerical computations have been carried out successfully for low values of $\mathrm{p}$ and $\mathrm{q}$ only (see sect.5). Several breakthroughs are due to James [13] and Constantine and Muirhead [14], as already mentioned. Contemporary research relies heavily on their results (see for example Bekker et al. [43]).

\subsubsection{Matrix-Transforms Approach}

Mathai [44] introduced the $M$-Transform method, which can establish several relations between hypergeometric functions, by using the fact that Laplace transforms are unique. It is based on the Weyl fractional integral, and a function $f(X)$ is, by definition, a $(r, s)$ - hypergeometric function, i.e.

$$
f(Z)={ }_{r} F_{s}\left(a_{1}, \cdots, a_{r} ; b_{1}, \cdots, b_{s} ; Z\right)
$$

if its $M$-transform, i.e. $\int_{Z=Z^{\prime}>0}|Z|^{\rho-\frac{m+1}{2}} \cdot f(-Z) \mathrm{d} Z$, is of the form

$$
\frac{\prod_{j=1}^{s} \Gamma_{m}\left(b_{j}\right) \prod_{j=1}^{r} \Gamma_{m}\left(a_{j}-\rho\right)}{\prod_{j=1}^{r} \Gamma_{m}\left(a_{j}\right) \prod_{j=1}^{s} \Gamma_{m}\left(b_{j}-\rho\right)} \Gamma_{m}(\rho) \text {, with } \rho \text { arbitrary such that the above expression on }
$$

gammas exists.

Similarly, the Lauricella function $F_{A}(\cdot)$ in $n$ matrix arguments can be defined as the function that can be represented as a $n$-fold integral, i.e. 


$$
\begin{aligned}
& F_{A}\left(a ; b_{1}, \cdots, b_{n} ; c_{1}, \cdots, c_{n} ; X_{1}, \cdots, X_{n}\right) \\
& =K \int_{0}^{I_{m}} \cdots \int_{0}^{I_{m}}\left|U_{1}\right|^{b_{1}-\frac{m+1}{2}} \cdots\left|U_{n}\right|^{b_{n}-\frac{m+1}{2}}\left|I_{m}-U_{1}\right|^{c_{1}-b_{1}-\frac{m+1}{2}} \cdots\left|I_{m}-U_{n}\right|^{c_{n}-b_{n}-\frac{m+1}{2}}\left|I_{m}-X_{1}^{1 / 2} U_{1} X_{1}^{1 / 2}-\cdots-X_{n}^{1 / 2} U_{n} X_{n}^{1 / 2}\right|^{-a} \mathrm{~d} U_{1} \cdots \mathrm{d} U_{n}, \\
& \text { where } K=\frac{\prod_{j=1}^{n} \Gamma_{m}\left(c_{j}\right)}{\prod_{j=1}^{n}\left\{\Gamma_{m}\left(b_{j}\right) \Gamma_{m}\left(c_{j}-b_{j}\right)\right\}} .
\end{aligned}
$$

Mathai [45] was able to define most hypergeometric functions of matrix arguments, including $H$ and $G$, with this approach, which is favored when we seek pure theoretical results only, since numerical computations seem quite difficult to undertake.

\subsection{Hypergeometric Function in Several Matrix Variates}

Hypergeometric function in two matrix variates is present in a basic result of multivariate analysis (Muirhead ([30], p.259)), defined with zonal polynomials, since it does not seem convenient, although possible, to define ${ }_{p} F_{q}(. ; X, Y)$ using either Laplace transform, or $M$-transform method.

$$
\int_{O(m)}{ }_{p} F_{q}\left(a_{1}, \cdots, a_{p} ; b_{1}, \cdots, b_{q} ; X H Y H^{\prime}\right)(\mathrm{d} H)={ }_{p} F_{q}^{(m)}\left(a_{1}, \cdots, a_{p} ; b_{1}, \cdots, b_{q} ; X, Y\right) \text {. }
$$

Here, $(\mathrm{d} H)$ is the normalized invariant measure on $O(m), X$ and $Y$ are symmetric $m \times m$ matrices, and

$$
{ }_{p} F_{q}^{(m)}\left(a_{1}, \cdots, a_{p} ; b_{1}, \cdots, b_{q} ; X, Y\right)=\sum_{k=0}^{\infty} \sum_{\kappa} \frac{\left(a_{1}\right)_{\kappa} \cdots\left(a_{p}\right)_{\kappa}}{\left(b_{1}\right)_{\kappa} \cdots\left(b_{q}\right)_{\kappa}} \frac{C_{\kappa}(X) C_{\kappa}(Y)}{k ! C_{\kappa}\left(I_{m}\right)} .
$$

It is straightforward to extend the number of matrices to $n>2$ (Mathai and Pederzoli [46]), even when using $H$ and $G$ functions.

\section{Computational Issues}

\subsection{Computation of the Hypergeometric Function}

In the past several serious efforts were made to find so-called computable forms for $H$ and $G$-functions, with some success since the formulas obtained are extremely complicated (see e.g. Mathai and Saxena [23]). Classical hypergeometric functions and $G$-functions, in one scalar variable, are now found in most commercial software (Maple, Mathematica, Matlab, etc.). In determining the numerical value of $G$ by MellinBarnes method, the number of poles can influence its accuracy, since this value is computed from the numerical values of residues at regular poles, as presented in Springer [26]. Pearson's thesis [47] discusses several points on the computation of ${ }_{2} F_{1}(\cdot)$. Table 17 there makes some recommendations on methods to be used. It is interesting to note here that, usually, the series $H S_{(2,1)}$ converges very slowly while the integral (5), or (5'), converges quite rapidly. Also, (5) and/or (5') remains valid when parameters differ by integers while (7) has to be adjusted. Section 2.3.1 above can be consulted for these questions. 
$G$-functions are used lately to carry out difficult definite integrals computations (Adamchik [48]) because of various relations that exist between transforms of $G$-functions, and between products of $G$-functions. For example,

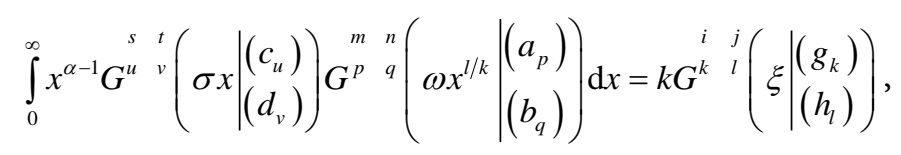

with the values of the parameters on the RHS obtainable from those of the LHS.

The two integral representations of $\mathrm{G}$ below are also used to deal with definite integrals:

$$
G^{p+1} q_{q+1}^{n+1}\left[\begin{array}{l}
\alpha, a_{1}, \cdots, a_{p} \\
b_{1}, \cdots, b_{q}, \beta
\end{array} ; z\right]=\frac{1}{\Gamma(\alpha-\beta)} \int_{1}^{\infty} x^{-\alpha}(1-x)^{\alpha-\beta-1} \cdot G^{m} \quad \begin{aligned}
& m \\
& q
\end{aligned}\left[\begin{array}{l}
a_{1}, \cdots, a_{p} \\
b_{1}, \cdots, b_{q}
\end{array} z x\right] \mathrm{d} x
$$

and

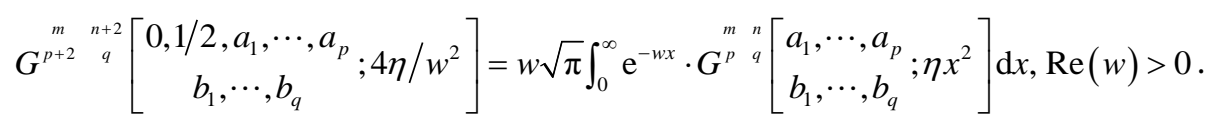

These properties have been used in the software on integration, called REDUCE (Gaskell [49], http://www.reduce-algebra.com/).

There are serious difficulties, however, in carrying out computations for hypergeometric functions in one or several matrix arguments, beginning with difficulties associated with zonal polynomials. Gutiérrez, Rodriguez and Saéz [50] are the early authors who reported results on this topic. Their work was limited to ${ }_{1} F_{1}(\cdot ; Z)$ and ${ }_{2} F_{1}(\cdot ; Z)$ and the values obtained from truncated series are quite good. However, there are already 627 zonal polynomials to be computed when $k=20$, demanding a lot of computer time. Koev and Edelman [51] have succeeded to have better accuracy and a much shorter computer time, by using Jack polynomials (which are generalizations of zonal polynomials), with an updating strategy to compute them. Butler and Wood [52], using the same Laplace approximation approach applied to one matrix argument in an earlier paper, reported fair to excellent accuracies in approximating ${ }_{p} F_{q}\left(\cdot ; Z_{1}, Z_{2}\right)$, for $p, q$ equal 0 or 1 .

The theory of Grobner basis has great influence on computations lately, in several domains of mathematics and algebraic statistics. Saito, Sturmfels and Nakayama [12] used it to study and approximate hypergeometric integrals belonging to the GKZ family. They also used it to study systems of multidimensional hypergeometric partial differential equations. This approach can be compared to the Perturbations approach to solve a problem in classical mathematics. There are several important results in [12] but they lie outside the scope of this survey.

As long as the computation of results cannot be made, progresses in that area are hampered. This is the case of zonal polynomials, which looked promising when they were first introduced, but there is now a high volume of highly complex theoretical results, and formulas, in need of confirmation by computation. Fortunately, fractional calculus applied to hypergeometric functions has some recent software and numerical methods recently made available (see Baleanu et al. [53], and the list therein of six hun- 
dred references).

New statistical technics are required in face of the data evolution. Now, the number of variables can be much larger than the sample size, as is frequently encountered in data sets in some statistical/biometric problems. Ledoit and Wolf's results [54] on estimating the covariance matrix in that case, are of interest. Similar approaches, related to other problems, are proposed by Fujikoshi and Ulyanov [55] in their joint work.

It should be mentioned that NIST, the National Institute of Standards and Technology (GB) maintains an on-line public library (Digital Library of Mathematical Functions at http:dlmf.nist.gov) with a special section on Functions of Matrix Argument.

\subsection{Old and New Relations between Hypergeometric Functions Managed by Computer}

It is understandable that the huge volume of relations between hypergeometric functions of all types presented in the literature, and new ones regularly introduced in journals, raise various pertinent questions: Are they correct? How can we recognize a series as being of hypergeometric type? Can some of them be merely modified versions of existing ones? What are the mechanisms to derive new results from existing fundamental ones? Can we identify those which are really basic?

Instead of manually consulting huge data bases of published results, different computer algorithms have been introduced, and run, to provide answers to the above questions. For example, Milgram [56] used computer algorithms to numerically test all closed forms ${ }_{3} F_{2}(1)$ identities given in Prudnikov et al. [57]. He could then omit some equations and amended others, as well as introduce a few new ones. By repeating this process he obtained a final of 89 identities, only 23 of them were in the original set (see Hannah [58] for other similar concepts and approaches).

\section{Hypergeometric Functions Derived via Other Approaches}

We have so far relied on infinite series and integrals to deal with hypergeometric functions in one scalar variable. Can it be done otherwise? Yes, and it can be derived from at least three other directions which differ drastically from the approaches starting with hypergeometric series (4) or (8). However, only the third one, the Carlson's approach, could be of immediate use in Statistics, in our opinion, the other two seem to be very advanced exercises to derive known or new results.

\subsection{Fractional Calculus}

Fractional calculus starts from the principle that a derivative can be of any order, unlike in classical calculus where these orders must be integers. Derivatives and integrals can then be unified into a single operation, called the differintegral: There are several approaches in defining a fractional derivative $D$ or integral $I$, the most popular one being the Riemann-Liouville integral,

$$
{ }_{a} I_{x}^{n} f(x)={ }_{a} D_{x}^{-n} f(x)=\int_{a}^{x} \int_{a}^{y_{1}} \cdots \int_{a}^{y_{n-1}} f\left(y_{n}\right) \mathrm{d} y_{n} \cdots \mathrm{d} y_{1}=\frac{1}{\Gamma(n)} \int_{a}^{x}(x-t)^{n-1} f(t) \mathrm{d} t,
$$


which leads to:

$$
{ }_{a} D_{t}^{\alpha} f(t)=\frac{\mathrm{d}^{n}}{\mathrm{~d} t^{n}}\left[{ }_{a} D_{t}^{-(n-\alpha)} f(t)\right]=\frac{\mathrm{d}^{n}}{\mathrm{~d} t^{n}}\left[{ }_{a} I_{t}^{(n-\alpha)} f(t)\right],
$$

with $n$ being the nearest integer larger than $\alpha$. With $\alpha>0$ we have a fractional derivative, and $\alpha<0$, a fractional integral (Kiryakova [59]).

Lavoie et al. [60] gives a simple survey of these approaches, mostly oriented toward special functions, which include Cauchy integral, Euler and Pochhammer contour integrals, etc. Leibnitz rule for derivatives of products becomes:

$$
D_{z}^{\alpha}[u(z) v(z)]=\sum_{n=-\infty}^{\infty}\left(\begin{array}{c}
\alpha \\
n+\gamma
\end{array}\right) D_{z}^{\alpha-n-\gamma} u(z) D_{z}^{n+\gamma} v(z) .
$$

The generalized hypergeometric function ${ }_{2} F_{1}$, expressed as a fractional derivative $D_{z}^{\gamma}$, is as follows:

$$
{ }_{2} F_{1}(a, b ; c ; z)=\frac{\Gamma(c)}{\Gamma(b)} z^{1-c} D_{z}^{b-c}\left[z^{b-1}(1-z)^{-a}\right],
$$

and the more general relation is:

$$
\begin{aligned}
& { }_{p+1} F_{q+1}\left[\begin{array}{l}
a_{1}, \cdots, a_{p}, c \\
b_{1}, \cdots, b_{q}, d
\end{array} \mid z\right] \\
& =\frac{\Gamma(d)}{\Gamma(c)} z^{1-d} D_{z}^{c-d}\left[z_{p}^{c-1} F_{q}\left[\begin{array}{c}
a_{1}, \cdots, a_{p} \\
b_{1}, \cdots, b_{q}
\end{array} \mid z\right]\right]^{\circ}
\end{aligned}
$$

Using fractional calculus, Kiryakova [59] shows that any special function is a differintegral of an elementary function. More precisely, we have 3 cases for ${ }_{p} F_{q}(\cdot)$ :

a) $p<q:{ }_{p} F_{q}(\cdot)$ is the differintegral of the generalized cosine function $\cos _{q-p+1}\left((q-p+1) x^{1 /(q-p+1)}\right)$.

b) $p=q:{ }_{p} F_{q}(\cdot)$ is the differintegral of the elementary function $x^{a_{1}-1} \mathrm{e}^{x}$.

c) $p=q+1:{ }_{p} F_{q}(\cdot)$ is the differintegral of the elementary function $x^{a_{2}-1}(1-x)^{-a_{1}}$.

Kiryakova [59] uses the Kober-Erdelyi transform with kernel the $G$-function, which is then shifted backwards from ${ }_{p} F_{q}(\cdot)$ to ${ }_{p-1} F_{q-1}(\cdot)$, and progressively to ${ }_{0} F_{q-p}(\cdot)$, to ${ }_{0} F_{0}(\cdot)$, or to ${ }_{0} F_{1}(\cdot)$ respectively. For example in the first case we have:

$$
\begin{aligned}
& { }_{p} F_{q}\left(a_{1}, \cdots, a_{p} ; b_{1}, \cdots, b_{q} ; x\right) \\
& =\left[\prod_{j=1}^{p} \frac{\Gamma\left(b_{q-p+j}\right)}{\Gamma\left(a_{j}\right)}\right] I_{1-p}^{\left(a_{k}-1\right),\left(b_{q-p+k}-a_{k}\right)}\left[{ }_{0} F_{q-p}\left(b_{1}, \cdots, b_{q-p} ; x\right)\right]^{.}
\end{aligned}
$$

Using Poisson type representation we obtain the cosine function.

$I_{a, b}^{c, d}[f(x)]$ is, however, a complicated generalized operator of fractional integration of Riemann-Liouville type,

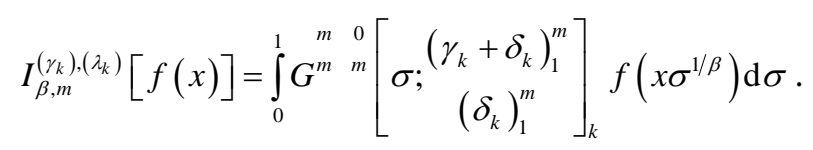


The generalized $\mathrm{m}$-tuple fractional derivative is then:

$$
D[f(x)]=x^{-\delta_{0}} D_{\beta, m}^{\left(\gamma_{k}-\left(\delta_{0} / \beta\right)\right),\left(\delta_{k}\right)}[f(x)],
$$

where

$$
D_{\beta, m}^{\left(\gamma_{k}\right),\left(\delta_{k}\right)}=\left[\prod_{r=1}^{m} \prod_{j=1}^{\eta_{r}}\left(\frac{1}{\beta} x \frac{\mathrm{d}}{\mathrm{d} x}+\gamma_{r}+j\right)\right] I_{\beta, m}^{\left(\gamma_{k}+\delta_{k}\right),\left(\eta_{k}-\delta_{k}\right)} .
$$

We have, as expected, $D_{\left(\beta_{k}\right), m}^{\left(\gamma_{k}\right),\left(\delta_{k}\right)} I_{\left(\beta_{k}\right), m}^{\left(\gamma_{k}\right),\left(\delta_{k}\right)}[f(x)]=f(x)$.

Using the composition of $\mathrm{m}$-tuple and $\mathrm{n}$-tuple integrals as $(m+n)$-tuple integral

$$
I_{\left(\beta_{k}\right), m}^{\left(\gamma_{k}+\delta_{k}\right),\left(\sigma_{k}\right)} I_{\left(\beta_{k}\right), m}^{\left(\gamma_{k}\right),\left(\delta_{k}\right)}[f(x)]=I_{\left(\beta_{k}\right), m}^{\left(\gamma_{k}\right),\left(\delta_{k}+\sigma_{k}\right)}, \quad k=1, \cdots, m, \delta_{k}, \sigma_{k}>0,
$$

and considering separately each of the three above cases, we obtain the above results.

NOTE: 1) This interesting result has to be interpreted with care however, since the special function $\mathrm{G}$ is used as kernel in the operator.

2) The idea of averaging, using simple functions, is similar to the one carried out by Carlson in (sect. 6.3) and other authors. Following the same idea, Pham-Gia [61] used the limit of an iterative convolution process to obtain interesting functions in quasi-analyticity.

There are several convincing applications of Fractional calculus in Engineering and Applied Probability. In Theoretical Statistics, several recent research results on hypergeometric functions use fractional calculus (Mathai [44]), associated with functions of matrix arguments (Mathai and Haubold [62]). But it is still too early to appraise the impact of this notion on Statistics.

\subsection{Lie Group Appproach}

Group theory has had important influence on Statistics. As stated by Giri [63], by introducing the group invariance principle and restricting attention to invariant decision rules a reduction of the dimension of the parametric space is possible. He also provides several examples where the hypergeometric functions are present. Group representation is another well-used concept in multivariate statistics, as seen in zonal polynomials. Wijsman [64] gives a simple example of how the distribution of $\chi_{v}$ can be obtained using this approach, and also some statistical problems to which a special group structure applies, called Type I.

It can be proved that, starting from the structure of an appropriate Lie Group, here the special linear group $S L(2, R)$, we can establish several properties of, and relations on, hypergeometric functions. Introduced in the late sixties by Miller Jr, among others, this approach seemed to be promising. It is based on the Lie group structure and the Lie Algebra which is the derivative at zero of the elements of the Lie group. The exponential function, using infinite series, permits to go from the Lie Algebra elements to the Lie group elements. Using a basis based on hypergeometric functions and commutators based on differential operators, several relations on hypergeometric functions can be derived. The following table (Wasson and Gilmore [65]) gives below the correspondence between the Lie group to be considered for the chosen special function. 


\begin{tabular}{cc} 
Lie Group & Special Function \\
Heisenberg group $H 3$ & Hermite polynomials \\
Euclidean group E2 & Bessel functions \\
Special unitary group $S U(2)$ & Legendre polynomials \\
& Jacobi Polynomials \\
Special linear group $S L(2, \mathbb{R})$ & Hypergeometric functions \\
Third order triangular matrices & Whittaker functions \\
& Laguerre polynomials \\
Special orthogonal group $S O(n)$ & Gegenbauer functions \\
\hline
\end{tabular}

Miller Jr [66] and Miller Jr [67] have presented the arguments concerning the two functions ${ }_{p} F_{q}(\cdot)$ and $G(\cdot)$. However, they are too lengthy to be reproduced here. But the main difficulties seem to be the selection of the Lie group to start with, and then the choice of these bases themselves, which can be quite complicated.

This highly mathematical approach is in the domain of theoretical mathematical physics, with few applications in Statistics. But the concept of symmetry frequently used here can be related to several symmetry problems in Statistics. Wijsman's monograph [64] is helpful in understanding several related abstract mathematical notions. Consequently, we have reservations that this approach can be used, in either Classical or Bayesian Statistics, although it is very elegant and seems helpful in establishing new relations for special functions. Lie group theory and Lie algebra have found some real applications, however, in Statistics on manifolds and on Image processing (see, for example, Fletcher, Lu and Joshi [68]).

\subsection{Carlson's Approach}

Carlson [10] introduced several hypergeometric functions of his own, which are different from the classical ones, e.g. $R_{n}(b, z)$ and $S(b, z)$ functions, which are obtained by averaging $x^{n}$ and $\exp (x)$, using a Dirichlet measure. The motivation is that expressions of $R_{n}(b, z)$ and $S(b, z)$ in the several complex variables domain are free of branch points, and can be better studied. Prof Carlson passed away quite recently.

Several notions developed here can be linked to the classical ones. For example, the so-called Euler measure is just the Lebesgue measure using the gamma density,

$$
\mathrm{d} \lambda_{\alpha}(t)=\frac{1}{\Gamma(\alpha)} t^{\alpha-1} \mathrm{e}^{-t} \mathrm{~d} t
$$

and the average derived

$$
\int_{0}^{\infty} F_{q}\left(a_{1}, \cdots, a_{p} ; b_{1}, \cdots, b_{q} ; t x\right) \mathrm{d} \lambda_{\alpha}(t)={ }_{p+1} F_{q}\left(\alpha, a_{1}, \cdots, a_{p} ; b_{1}, \cdots, b_{q} ; x\right)
$$

is our relation (10) above.

According to Carlson [10], $R_{n}(b, z)$ is supposed to play several roles, those of ${ }_{2} F_{1}$, those of the elliptic integral and those of Appell's $F_{1}(\cdot)$, while the couple $\left\{S(b, z),{ }_{0} F_{2}(\cdot)\right\}$ replaces ${ }_{1} F_{1}(\cdot)$. 
We have, in particular:

$$
{ }_{2} F_{0}(\alpha, \beta ; x)=\int_{0}^{\infty} \int_{0}^{\infty} \mathrm{e}^{s t x} \mathrm{~d} \lambda_{\alpha}(s) \mathrm{d} \lambda_{\beta}(t) .
$$

Several classical special functions can be shown to be particular cases of $R_{n}(b, z)$ and hence, are Dirichlet averages of elementary functions. Even the Schwarz-Christoffel mapping in complex analysis can be shown to be an $R_{n}(b, z)$-function too.

\subsubsection{Definitions of Functions $R_{n}(b, z)$ and $S(b, z)$ as Averages}

Using a general averaging process with a Dirichlet distribution on a simplex $\Delta$, we define:

$$
\mathrm{d} \mu_{b}=\frac{1}{B(b)} u_{1}^{b_{1}-1} \cdots u_{k-1}^{b_{k-1}-1}\left(1-u_{1}-\cdots-u_{k-1}\right)^{b_{k}-1} \mathrm{~d} u_{1} \cdots \mathrm{d} u_{k-1},
$$

with

$$
\Delta=\left\{\left(u_{1}, \cdots, u_{k-1}\right), 0 \leq u_{i} \leq 1,0 \leq \sum_{i-1}^{k-1} u_{i} \leq 1\right\} .
$$

For any measurable function $f$, we define the average of $f$ w.r.t. a Dirichlet measure $\mu_{b}$ as

$$
F(b, z)=\int_{E} f(u \cdot z) \mathrm{d} \mu_{b}(u), \quad z=\left(z_{1}, \cdots, z_{k}\right) \in \Omega^{k}, u \cdot z=\sum_{i=1}^{k} u_{i} z_{i} .
$$

Here, $\Omega$ is a convex set in $\mathbb{C}$ and $E$ is the standard simplex in $\mathbb{R}^{k-1}$.

Hence, the averages w.r.t. power functions, $z \in C \mathbb{C}^{k}$, is:

1) $R_{n}(b, z)=\int_{\Delta}(u \cdot z)^{n} \mathrm{~d} \mu_{b}(u), n \in \mathbb{N}$, and

2) $R_{t}(b, z)=\int_{\Delta}(u \cdot z)^{t} \mathrm{~d} \mu_{b}(u), \quad t \in \mathbb{C}-\mathbb{N}$.

Similarly, the average w.r.t. to the exponential is

3) $S(b, z)=\int_{\Delta} \mathrm{e}^{u \cdot z} \mathrm{~d} \mu_{b}(u)$.

\subsubsection{Results of Interest}

1) There are several relations between these functions, and with the classical hypergeometric functions. In fact, $R_{n}(\cdot)$ can be expressed as a polynomial

$$
R_{n}(b, z)=\sum_{|m|=n} \frac{n !}{(c, n)} \frac{\left(b_{1}, m_{1}\right) \cdots\left(b_{k}, m_{k}\right)}{m_{1} ! \cdots m_{k} !} z_{1}^{m_{1}} \cdots z_{k}^{m_{k}} .
$$

2) Relations between $R_{n}(b, z)$ and ${ }_{2} F_{1}$ and $F_{D}$ :

a) ${ }_{2} F_{1}(\alpha, \beta ; \gamma ; x)=R_{-\alpha}(\beta, \gamma-\beta ; 1-x, 1)$.

$$
R_{n}\left(a ; b_{1}, \cdots, b_{n} ; z_{1}, \cdots, z_{n}\right)
$$

b) $=F_{D}^{(n)}\left(a ; b_{1}, \cdots, b_{n} ; b_{1}+\cdots+b_{n} ; 1-z_{1}, \cdots, 1-z_{n}\right)$

$$
=z^{-a} F_{D}^{(n-1)}\left(a ; b_{1}, \cdots, b_{n-1} ; b_{1}+\cdots+b_{n} ; 1-z_{1} / z_{n}, \cdots, 1-z_{n-1} / z_{n}\right) .
$$

c) Several other relations relating $R_{n}(b, z)$ to ${ }_{2} F_{1}, S$ and ${ }_{1} F_{1}$ exist (see Carlson 
[10]).

\subsubsection{Single Integral Representation and Elliptic Integrals}

1) Representation by a single integral: $R_{-a}(b, z)$, which is a multiple integral, can be reduced to a single integral on $[0,1]$, i.e.

$$
R_{-a}(b, z)=\int_{0}^{1} \prod_{i=1}^{k}\left(1-u+u z_{i}\right)^{-b_{i}} \mathrm{~d} \mu_{\alpha}(u)
$$

where $\mu_{\alpha}$ is a beta measure on $[0,1]$, with $\alpha=\left(a, a^{\prime}\right), a+a^{\prime}=\sum_{i=1}^{k} b_{i}$.

This single integral gives the holomorphic continuation of $R_{-a}(b, z)$ in $C^{k}$.

2) Connections between Appell function $F_{1}(\cdot)$ and elliptic integrals: They are found by Carlson [10].

A particular case of the hypergeometric integral considered in section 8.2, in a theoretical context, is the elliptic integral

$$
I=\int_{0}^{\infty}\left[(x+A)^{2}(x+B)^{2}(x+C)^{2}(x+D)^{2}\right]^{-1 / 2} \mathrm{~d} x
$$

that can be now shown to be equal to

$$
\int_{0}^{\infty}\left[\left(t+E^{2}\right)\left(t+F^{2}\right)\left(t+G^{2}\right)\right]^{-1 / 2} \mathrm{~d} t
$$

where $E=A B+C D, F=A C+B D, G=A D+B C$.

Furthermore, setting:

$$
\left\{\begin{array}{l}
(x y z)^{-1 / 2}=R_{-3 / 2}(1 / 2,1 / 2,1 / 2 ; x, y, z) \\
R_{F}(x, y, z)=R_{-1 / 2}(1 / 2,1 / 2,1 / 2 ; x, y, z) \\
R_{G}(x, y, z)=R_{1 / 2}(1 / 2,1 / 2,1 / 2 ; x, y, z) \\
R_{H}(x, y, z, \rho)=R_{-1 / 2}(1 / 2,1 / 2,1 / 2,1 ; x, y, z, \rho)
\end{array}\right.
$$

we now have $I=2 R_{F}\left(E^{2}, F^{2}, G^{2}\right)$, which is a very convenient symmetrical form.

Carlson's various hypergeometric functions are found to be quite useful by Askey [9] and have seen several applications in Bayesian Statistics (Dickey [69] and in the theory of elliptic functions (Carlson [70]).

\subsection{Basic Q-Hypergeometric Functions}

There is a parallel theory of hypergeometric functions based on q-hypergeometric series. Here, the ratios of successive terms are a rational function of $q^{n}$. We then have:

$$
(\lambda ; q)_{n}=\left\{\begin{array}{l}
1, n=0 \\
(1-\lambda)(1-\lambda q)\left(1-\lambda q^{2}\right) \cdots\left(1-\lambda q^{n-1}\right), n=1,2,3, \cdots
\end{array}\right.
$$

for any $q, \lambda$, real or complex, $|q|<1$, and the corresponding q-basic hypergeometric series is:

$$
{ }_{r} \Phi_{s}^{(n)}\left(a_{1}, \cdots, a_{r}, ; b_{1}, \cdots, b_{s} ; q ; z\right)=\sum_{n=0}^{\infty}\left[(-1)^{n} q^{n(n+1) / 2}\right]^{1+s-r} \frac{\left(a_{1}, q\right)_{n} \cdots\left(a_{r}, q\right)_{n}}{\left(b_{1}, q\right)_{n} \cdots\left(b_{s}, q\right)_{n}} \frac{z^{n}}{(q, q)_{n}} .
$$

Several results here are similar to the ones we have seen, but some are quite different. 
We will not discuss this approach further and refer the reader to Srivastava and Karllson [29]. It should be mentioned that Ramanujan has established several interesting results in this domain.

\section{Presence of Hypergeometric Functions in Statistics}

As stated earlier, in Statistics, Hypergeometric functions are generally not developed, but used, and mostly in distribution theory.

\subsection{Discrete Case}

Hypergeometric distribution in unidimensional statistics:

a) There are $X$ "good" elements in a population of $N$. The probability of having $X$ "good" when choosing at random $n$ elements is (in finite sampling without replacement):

$$
\operatorname{Pr}(X=x)=\left(\begin{array}{c}
X \\
x
\end{array}\right)\left(\begin{array}{c}
N-X \\
n-x
\end{array}\right) /\left(\begin{array}{c}
N \\
n
\end{array}\right), \quad \max (0, n-N+X) \leq x \leq \min (n, X) .
$$

The moment generating function of this distribution is

$$
E\left(\mathrm{e}^{t X}\right)=\frac{(N-n)(N-X)}{N !} \cdot{ }_{2} F_{1}\left(-n,-X, N-X-n+1 ; \mathrm{e}^{t}\right) .
$$

This fact gives this discrete distribution its name. It must be mentioned that it is the conditional distribution, on which Fisher's exact test on proportions is based.

b) A generalization of this distribution leads to the Kemp family, which is based on a generalization of the above probability, i.e.

$$
\operatorname{Pr}(X=r)=\left(\begin{array}{c}
a \\
r
\end{array}\right)\left(\begin{array}{c}
b \\
n-r
\end{array}\right) /\left(\begin{array}{c}
a+b \\
n
\end{array}\right), r=0,1,2, \cdots,
$$

for arbitrary positive values of $a$ and $b$. Several types of distributions are obtained and reported in Johnson and Kotz ([71], chapter 6). Derived distributions include the Non-Central Hypergeometric distribution, and the related positive and negative hypergeometric distributions.

The discrete multivariate hypergeometric distribution is a straightforward extension of the univariate case: Instead of one good subset we have $(k-1)$ distinct good ones and the $k$-th subset is the bad ones. For a sample of size $m$, and $X_{1}=x_{1}, \cdots, X_{k-1}=x_{k-1}$, with $X_{k}=x_{k}=m-\sum_{j=1}^{k-1} x_{j}$ we have:

$$
\operatorname{Pr}\left(X_{1}=x_{1}, \cdots, X_{k-1}=x_{k-1}\right)=\left\{\prod_{i=1}^{k}\left(\begin{array}{c}
M_{i} \\
x_{i}
\end{array}\right)\right\} /\left(\begin{array}{c}
M \\
x
\end{array}\right), \quad M=\sum_{i=1}^{k} M_{i}, \quad x=\sum_{i=1}^{k} x_{i} .
$$

We refer to chapter 39 of Johnson, Kotz and Balakrishnan [72] for other properties of this discrete multivariate distribution.

\subsection{Continuous Case}

a) Gauss hypergeometric function ${ }_{2} F_{1}(\cdot)$ has found applications mostly in distribution theory (e.g. Pham-Gia and Turkkan [73] and [74]). 
A nice property of hypergeometric functions, especially ${ }_{1} F_{1}$ and ${ }_{1} F_{2}$, is that they could provide, by mere multiplication with the central density, the expression of the non-central density. For example, the density of the central $F, \quad X \sim F_{v_{1}, v_{2}}$, defined here as the ratio of two independent chi-square $F_{v_{1}, v_{2}}=\frac{\chi_{v_{1}}^{2}}{\chi_{v_{2}}^{2}}$, is $h_{F}\left(v_{1}, v_{2} ; f\right)=\frac{1}{B\left(v_{1} / 2, v_{2} / 2\right)} \frac{f^{\frac{v_{1}}{2}-1}}{\left(1+\frac{v_{1}}{v_{2}} f\right)^{\frac{v_{1}+v_{2}}{2}}}$.

The related non-central variable $G$, with non-centrality parameter $\lambda, G \approx F_{v_{1}, v_{2}, \lambda}$, will have as density

$$
h_{G}\left(v_{1}, v_{2} ; \lambda ; g\right)=h_{G}\left(v_{1}, v_{2} ; g\right) \cdot \mathrm{e}^{-\lambda / 2} \cdot{ }_{1} F_{1}\left(\frac{v_{1}+v_{2}}{2} ; \frac{v_{1}}{2} ; \frac{\lambda g}{2(1+g)}\right) .
$$

This fact is particularly useful when we study the power of a test, which uses the non-central distribution of a statistic. If we define $F_{v_{1}, v_{2}}=\frac{\chi_{v_{1}}^{2} / v_{1}}{\chi_{v_{2}}^{2} / v_{2}}$, we have similar results relating $h_{F}\left(v_{1}, v_{2} ; f\right)$ to $h_{G}\left(v_{1}, v_{2} ; \lambda ; g\right)$. A similar result holds for the noncentral beta distribution. Pham-Gia and Turkkan [36] provides the comparison between ratios of random variables and ratios of random matrices, and hypergeometric functions of various types are used in both cases.

$G$ and $H$-functions are used in the expressions of the densities of several positive random variables and in the distributions of determinants of random matrices, as shown by Pham-Gia [75]. The following variables with their densities limited to their positive part, have their density expressed as a $G$ or $H$-function: the half- standard normal, the half-Cauchy, the half-Student $t$ (Springer [26], pp. 202-207). The Cumulative Distribution Function of a $H$-function density variable is also expressible as a $H$-function, and so are its Laplace transform and characteristic function.

When considering a random Beta matrix variate, its determinant has its density expressed as a $G$-function since it is a product of independent univariate betas, and so do products and ratios of independent random matrices and several test statistics in multivariate analysis (e.g. Pham-Gia and Choulakian [76], Rathie [27]). The three types of $\begin{array}{llllll}m & 0 & m & 0 & m & n\end{array}$ $G$-functions mostly encountered here are: $G^{m}{ }^{m}, G^{0}{ }^{m}, G^{p}{ }^{q}$. But, as Mathai and Saxena ([23], sect 5.6) have remarked, often we have here the cases where the parameters differ by integers and computations of residues have to be adjusted accordingly.

b) Relations between hypergeometric functions and the normal distribution: What are the relations between these two most important notions in Statistics?

We have already mentioned the half-standard normal density expressed as a $G$-function. And an interesting relation exists on moments. Let $X \sim N\left(\mu, \sigma^{2}\right)$. We then have the raw moments: 


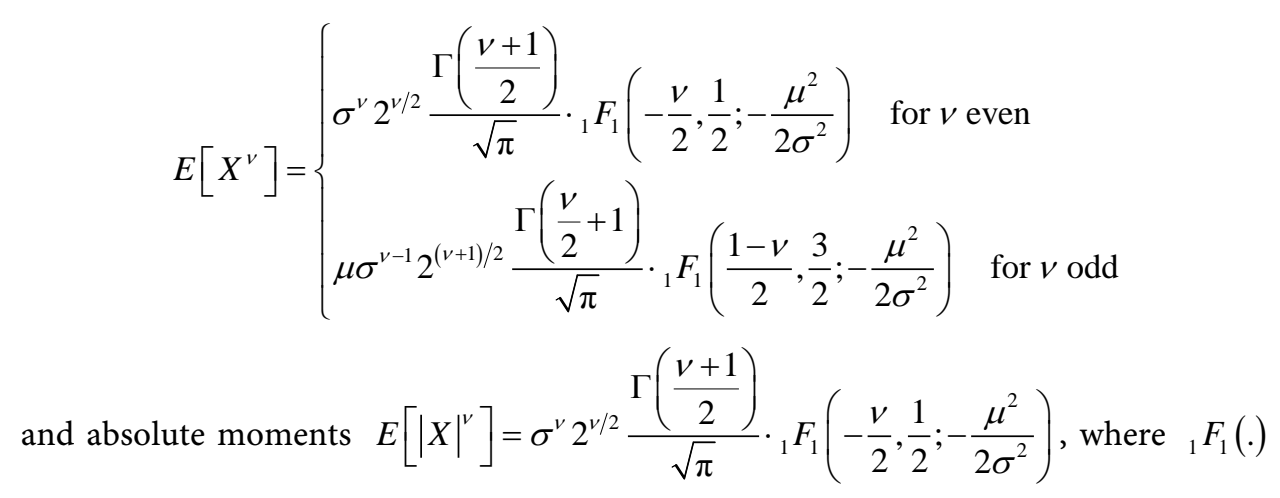
is Kummer confluent hypergeometric function. Central moments have, however, simple expressions: $E\left[(X-\mu)^{v}\right]=\left\{\begin{array}{ll}\sigma^{2 k}(2 k) ! /\left(k ! 2^{k}\right) & \text { for } v \text { even }=2 k \\ 0 & \text { for } v \text { odd }\end{array}\right.$, and $E\left(|X-\mu|^{v}\right)=\sigma^{v} 2^{v / 2} \frac{\Gamma\left(\frac{v+1}{2}\right)}{\sqrt{\pi}}$.

Here, again, we can see that ${ }_{1} F_{1}($.$) is associated with the non-centrality factor.$

\subsection{Matrix Case}

We have already mentioned the works of James [13] and Constantine and Muirhead [14] on zonal polynomials. Farrell [77], Pillai [78] and Olkin and Rubin [79] also made significant contributions. For functions of matrix arguments there are several results where these functions are associated with fractional calculus, under various forms (see Mathai and Haubold [62]), most of them still at the very theoretical level, however. They will probably make an impact on statistics in the years ahead. An application of interest is given by Gross and Richards [80].

\subsection{Other Applications}

Handbook of the beta distribution (see Gupta and Nadarajah [81]) has a selection of articles containing various hypergeometric functions in one or two scalar variables. In particular, Pham-Gia, in that reference, and Pham-Gia and Turkkan [82] has hypergeometric functions applications in Bayes inference. Exton ([28], chapter 7) and Mathai and Saxena [23] should be consulted for a large list of applications of multivariate hypergeometric functions in Statistics. Hypergeometric functions of matrix arguments are encountered in non-central matrix distributions and in power calculation for hypothesis testing involving vector and matrix variates. Mathai [44] and Mathai and Pederzoli [46] offered several theoretical results on this topic. Applications of functions with matrix arguments in engineering include Chiani et al. [83], Gross and Richards [80] and Tulino and Verdu [84].

\section{Hypergeometric Functions in Neighboring Domains}

Hypergeometric functions, being special mathematical functions, are traditionally associated with classical mathematical analysis, recurrence formulas and other special func- 
tions. An interesting account of their history is given by Stephen Wolfram [85]. But, for special functions, the establishment of numerous mathematical relations relating them via classical analysis methods, seems to lack some mathematical depth (Aomoto and Kita [86]). Using complex analysis, homological algebra, and algebraic and differential geometries, together with other abstract advanced mathematical techniques, some impressive results were obtained in the study of more in-depth mathematical properties of hypergeometric functions. Notions such as holonomic functions, monodromy groups, are frequently used, but we will not present them here since they are very seldom encountered in Statistics. Again, below are just some results, among so many others, that we think could be of interest to statisticians.

\subsection{Algebraic Topology, Algebraic K-Theory, Algebraic Geometry}

Hypergeometric integrals are the main concern of these fields, in which some important results can be presented under.

\subsubsection{Integral Representations}

We define first the Hypergeometric series of type $(n+1, m+1)$ : Let us consider the power series

$$
F\left(\left(\alpha_{i}\right),\left(\beta_{i}\right), \gamma, x\right)=\sum_{v} \frac{\prod_{i=1}^{n}\left(\alpha_{i} ; a_{i}(v)\right) \sum_{j=1}^{m-n-1}\left(\beta_{j} ; b_{j}(v)\right)}{(\gamma ; c(v)) v !} x^{v}
$$

defined by the lattice formed by the set $M_{n, m-n-1}(Z)$ of matrices $n \times(m-n-1)$ with integral coefficients and $m$ linear forms

$$
\begin{aligned}
& a_{i}(v)=\sum_{j=1}^{m-n-1} v_{i j}, 1 \leq i \leq n . \text { Naturally, the notation is: } \\
& x^{v}=\prod x_{i j}^{v_{i j}}, v !=\prod v_{i j} !, b_{j}(v)=\sum_{i=1}^{n} v_{i j}, 1 \leq j \leq m-n-1, \\
& c(v)=\sum_{i=1}^{n} \sum_{j=1}^{m-n-1} v_{i j}, v=\left(v_{i j}\right) \in M_{n, m-n-1}(Z)
\end{aligned}
$$

We can see that Gauss ${ }_{2} F_{1}(\cdot)$, Appell's $F_{1}(\cdot)$ and Lauricella $F_{D}(\cdot)$ are of this type. They have 2 integral representations, as stated in Theorem 3.3 of Aomoto and Kita [86],

a)

$$
F\left(\left(\alpha_{i}\right),\left(\beta_{i}\right) ; \gamma ; x\right)=c_{1} \int_{\Delta^{n}\left(\omega_{1}\right)} U_{1}(u) \cdot \prod_{j=1}^{m-n-1}\left(1-x_{1 j} u_{1}-\cdots-x_{n j} u_{n}\right)^{-\beta_{j}} \mathrm{~d}^{n} u
$$

with

$$
c_{1}=\frac{\Gamma(\gamma)}{\Gamma\left(\gamma-\sum_{i=1}^{n} \alpha_{i}\right)} \prod_{i=1}^{n} \Gamma\left(\alpha_{i}\right) \text { and } U_{1}(u)=\prod_{i=1}^{n} u_{i}^{\alpha_{i}-1}\left(1-u_{1}-\cdots-u_{n}\right)^{\gamma-\sum \alpha_{i}-1} .
$$

b)

$$
F\left(\left(\alpha_{i}\right),\left(\beta_{i}\right) ; \gamma ; x\right)=c_{2} \int_{\Delta^{m-n-1}\left(\omega_{2}\right)} U_{2}(u) \cdot \prod_{j=1}^{n}\left(1-x_{i 1} u_{1}-\cdots-x_{i, m-n-1} u_{m-n-1}\right)^{-\alpha_{i}} \mathrm{~d}^{m-n-1} u,
$$


with

$$
c_{2}=\frac{\Gamma(\gamma)}{\Gamma\left(\gamma-\sum_{j=1}^{m-n-1} \beta_{j}\right)} \prod_{j=1}^{m-n-1} \Gamma\left(\beta_{j}\right) \text { and } U_{2}(u)=\prod_{j=1}^{m-n-1} u_{j}^{\beta_{j}-1}\left(1-u_{1}-\cdots-u_{m-n-1}\right)^{\gamma-\sum \beta_{j}-1} .
$$

Similarly, Aomoto and Kita [86] show that an integral representation is possible for Horn's 14 hypergeometric series.

\subsubsection{Single Integral Representation}

This topic is related to the preceding one, and has attracted attention for a long time, since integrating in one variable is supposedly much simpler than doing it in several ones. There are at least three known cases, and we start with the Dirichlet distribution, $b\left(b_{1}, \cdots, b_{p+q}\right)$, defined on a simplex, of which the univariate beta $\beta\left(\beta_{1}, \beta_{2}\right)$, defined on $[0,1]$, is a special case.

1) Let $p, q$ be positive integers and $\mu_{b}, \mu_{\beta}$ Dirichlet and beta measures where $\beta=\left(\beta_{1}=b_{1}+\cdots+b_{p}, \beta_{2}=b_{p+1}+\cdots+b_{p+q}\right)$ on $E_{p+q-1}$ and $E_{1}$ resp. Let $\varphi(\cdot)$ be continuous, complex-valued on $[0,1]$. Then

$$
\int_{E_{p+q-1}} \varphi\left(v_{1}+\cdots+v_{p}\right) \mathrm{d} \mu_{b}(v)=\int_{0}^{1} \varphi(u) \mathrm{d} \mu_{\beta}(u) .
$$

Also,

$$
\int_{E_{p}} \varphi\left(v_{1}+\cdots+v_{p}\right) v_{1}^{b_{1}-1} \cdots v_{p}^{b_{p}-1} \mathrm{~d} v_{1} \cdots \mathrm{d} v_{p}=B\left(b_{1}, \cdots, b_{p}\right) \int_{0}^{1} u^{b_{1}+\cdots+b_{p}-1} \varphi(u) \mathrm{d} u .
$$

2) Carlson $R_{-a}(b, z)$ can be expressed as a single integral, in Equation (36).

3) For hypergeometric functions, Picard's Theorem (Equation (19)) on $F_{D}(\cdot)$, already mentioned, expresses this function as a single integral.

4) Here, we can see that Picard's integral is a particular case of Equation (41) above, when $n=1$. Equation (41) itself, is hence a generalization of Picard's theorem to the case $n>1$, using the appropriate simplex.

\subsubsection{A-Hypergeometric Functions}

In the late eighties, Gelfand, Kapranov and Zelevinsky considered all the vector generalizations of Gauss hypergeometric functions, and the related differential equations, and fit them into the system of A-hypergeometric functions.

A GKZ (Gelfand, Kapranov, Zelevinsky) hypergeometric system is recently renamed $A$-hypergeometric system. It starts with an $A$-Matrix, $r \times N$ with columns $a_{1}, \cdots, a_{N}$, hence its name, and is defined as follows (Saito et al. [12], p. 49): Let $A=\left(a_{i j}\right)$ be an integer $d \times n$ matrix of rank $d$ and let $\beta=\left(\beta_{1}, \cdots, \beta_{d}\right)$ be a vector of parameters. The GKZ system $H_{A}(\beta)$ is the system of linear partial differential equations for an indeterminate function $f$, such that:

$$
\begin{aligned}
& \left(\sum_{j=1}^{n} a_{i j} x_{j} \partial_{j}-\beta_{i}\right) \cdot f=0, i=1, \cdots, d \\
& \left(\partial^{u}-\partial^{v}\right) \cdot f=0, u, v \in N^{n}, A u=A v .
\end{aligned}
$$


For hypergeometric functions we assume that the last row of $A$ is constant, i.e. $a_{d 1}=a_{d 2}=\cdots=a_{d n}=p$ and let $\alpha, \gamma_{1}, \cdots, \gamma_{d-1}$ be integers. We set $\gamma_{d}=-p \alpha$, $n=d-1, \quad \beta=\left(-\gamma_{1}-1, \cdots,-\gamma_{d-1}-1,-\gamma_{d}\right)^{\mathrm{T}}$. Then $H_{A}(\beta)$ annihilates the hypergeometric integral

$$
\Phi(\gamma ; x)=\int_{C}\left(\sum_{i=1}^{n} x_{i} t^{a_{i}}\right)^{\alpha} t^{\gamma} \mathrm{d} t_{1} \cdots \mathrm{d} t_{n}
$$

(hence this integral satisfies the GKZ hypergeometric system).

A solution for the above system can be investigated under the form of a multiple series of the following form, which include most series in section 3 .

$$
\Psi_{L, \gamma}\left(u_{1}, \cdots, u_{n}\right)=\sum_{\left(l_{1}, \cdots, l_{n}\right) \in L} \frac{u_{1}^{l_{1}+\gamma_{1}}}{\Gamma\left(l_{1}+\gamma_{1}+1\right)} \cdots \frac{u_{n}^{l_{n}+\gamma_{n}}}{\Gamma\left(l_{n}+\gamma_{n}+1\right)} .
$$

We can verify that Gauss hypergeometric function ${ }_{2} F_{1}(x)$, Appell first hypergeometric function $F_{1}(x, y)$ and Horn $G_{3}(x, y)$ have this form (Beukers [87]), by choosing the appropriate $A$-matrix which determines the polytope, the vector-parameters $\gamma, \beta$, and $\gamma$, and setting all variables other than $x$, or $x$ and $y$, as 1. Important results in the field of several complex variables are obtained by using this approach.

It should be mentioned that there are several applications in Combinatorics of $A$-hypergeometric functions, for example in arranging a number of hyperplanes in a multi-dimension complex space.

\subsection{Hypergeometric Integrals in Conformal Field Theory, Homology and Cohomology}

a) Varchenko [88] considers the integral

$$
I(\Delta, f, \alpha)=\int_{\Delta} f_{1}^{\alpha_{1}} \cdots f_{N}^{\alpha_{N}} \mathrm{~d} x_{1} \cdots \mathrm{d} x_{N},
$$

and later, the more general form:

$$
I(\Delta, f, \alpha)=\int_{\Delta} \exp (x) f_{1}^{\alpha_{1}} \cdots f_{N}^{\alpha_{N}} \mathrm{~d} x_{1} \cdots \mathrm{d} x_{N},
$$

where $\Delta$, the polytope, is now a variable also, $f_{i}$ are linear functions and $\alpha_{i}$ are complex numbers. They are also called hypergeometric integrals and generalize the beta function. An interesting example is a configuration of 3 consecutive points $z_{1}, z_{2}, z_{3}$ on a line. Put $\Delta_{1}, \Delta_{2}$ as the consecutive distances separating them, and

$$
f_{j}=t-z_{j}, \quad l_{\alpha}=\left(t-z_{1}\right)^{\alpha_{1}}\left(t-z_{2}\right)^{\alpha_{2}}\left(t-z_{3}\right)^{\alpha_{3}}, \omega_{1}=\alpha_{1} l_{\alpha} \frac{d\left(t-z_{1}\right)}{t-z_{1}}, \omega_{2}=\alpha_{2} l_{\alpha} \frac{d\left(t-z_{2}\right)}{t-z_{2}} \text {. }
$$

Then we have:

$$
\operatorname{det}\left(\int_{\Delta_{i}} \omega_{j}\right)=\frac{\prod_{j=1}^{3} \Gamma\left(\alpha_{j}+1\right)}{\Gamma\left(\sum_{j=1}^{3} \alpha_{j}+1\right)} \prod_{i \neq j} f_{i}^{\alpha_{i}}\left(z_{j}\right)
$$

meaning that the determinant of integrals of hypergeometric forms of a configuration, 
over all bounded components of the complement of that configuration, can be simply computed. This formula can be extended to a configuration of hyperplanes.

There are several important results on hypergeometric functions in Conformal Field theory, on representation theory of Lie Algebra, in quantum groups, etc. However, they do not fit into this survey and the reader is invited to consult Varchenko [88]. For example, the integral

$$
I\left(t_{1}, \cdots, t_{n}\right)=\int \prod_{1 \leq j<k \leq N}\left(t_{j}-t_{k}\right)^{\lambda_{j k}} \mathrm{~d} t_{n+1} \cdots \mathrm{d} t_{N}, N=m+n
$$

can be interpreted as average of interactions of the last $m$ points with the first $n$ points, and can be shown to be associated with a representation of Kac-Moody algebra.

b) An interesting point of view can be taken for hypergeometric integrals, using the fact that definite integrals are considered as pairings of homology and cohomology groups according to de Rham Theory.

Let $T$ be an $m$-dimension complex manifold, or equivalently, as a $2 m$-dimension real smooth manifold. Let $\sigma_{i}$ be a smooth map from the $p$-dim simplex to $T$. A finite sum $C=\sum c_{i} \sigma_{i}, \quad c_{i} \in C$, is called a $p$-chain, and a $p$-cycle if $\partial C=\sum c_{i} \partial \sigma_{i}=0$, where $\partial$ is the boundary operator.

The homology group $H_{p}(T, C)$ is vector space of $p$-cycles modulo (Image of $(p+1)$ chains by operator $\partial)$. Let's consider the de Rham cohomology group $H^{p}(T, C)$ which is the quotient space:

$$
\frac{\{C \text {-valued smooth } p \text {-forms } \omega \text { s.t. } \mathrm{d} \omega=0\}}{d\{C \text {-valued smooth }(p-1) \text {-forms }\}} .
$$

We know that there is an isomorphism: $H^{p}(T, C) \simeq H_{p}(T, C)$. By Stokes Theorem

$$
\int_{\partial C} \omega=\int_{C} \mathrm{~d} \omega .
$$

We define $\langle C, \omega\rangle=\int_{C} \omega$, which leads to a bilinear form:

$\langle. ;\rangle:. H_{p}(T, C) \times H^{p}(T, C) \rightarrow C$.

The GKZ or A-hypergeometric integral is

$$
\Phi(\gamma, x)=\int_{C} f(x, t)^{\alpha} t^{\gamma} \mathrm{d} t_{1} \cdots \mathrm{d} t_{m} \text {, with } f(x, t)^{\alpha}=\sum_{i=1}^{n} x_{i} t_{1}^{\alpha_{1 i}} \cdots t_{d}^{\alpha_{d i}},
$$

where $t=\left(t_{1}, \cdots, t_{m}\right)$, and similarly for $x, \alpha, \gamma$.

We can see that the hypergeometric integral is a pairing between homology groups and cohomology groups, with its value being a function of $x$. A simple illustration using $p=1$, is the winding number in complex analysis, which is a pairing between $H^{1}(T, C)$ and $H_{1}(T, C)$. It satisfies the GKZ-hypergeometric system as presented above. This theoretical result is of importance although it does not permit to calculate the value of the integral.

For complex variables we have twisted homology and cohomology, as explained in Aomoto and Kita [86]. 


\subsection{Algebraic Functions and Roots of Equations}

Hypergeometric functions have been used to find solutions of algebraic equations of fifth order and higher. The reason is that its expression as an infinite series can be conveniently used for the search for a solution. For example, with the equation: $a x^{5}-x+1=0$ we can use Lagrange inversion formula that states that one solution to $f(z)=a$ is given by the power series

$$
\sum_{k=1}^{\infty} \frac{a^{k}}{k !}\left(\frac{\mathrm{d}}{\mathrm{d} x}\right)^{k-1}\left(\frac{x}{f(x)}\right)_{x=0}^{k}
$$

Here, we have:

$$
\left(\begin{array}{c}
5 n \\
n
\end{array}\right)\left(\frac{a^{n}}{4 n+1}\right)=\frac{5^{5 n}}{4^{4 n}} \frac{(1 / 5)_{n}(2 / 5)_{n}(3 / 5)_{n}(4 / 5)_{n}}{(1 / 2)_{n}(3 / 4)_{n}(5 / 4)_{n}} \frac{a^{n}}{n !} .
$$

Setting

$$
{ }_{4} F_{3}(1 / 5,2 / 5,3 / 5,4 / 5 ; 1 / 2,3 / 4,5 / 4 ; z)=\sum_{n=0}^{\infty} \frac{(1 / 5)_{n}(2 / 5)_{n}(3 / 5)_{n}(4 / 5)_{n}}{(1 / 2)_{n}(3 / 4)_{n}(5 / 4)_{n}} \frac{z^{n}}{n !}
$$

we have the solution of the equation as the hypergeometric function

$$
{ }_{4} F_{3}\left(1 / 5,2 / 5,3 / 5,4 / 5 ; 1 / 2,3 / 4,5 / 4 ; \frac{5^{5}}{4^{4}} a\right) .
$$

There is a classification list by H.A. Schwarz, of hypergeometric functions which are at the same time algebraic (Beukers [87]). This list has been recently extended by Beukers and Heckman [89]. Perelomov [90] gives hypergeometric solutions to more general algebraic equations.

\subsection{Economics, Quantitative Economics and Econometrics}

It is not surprising that hypergeometric functions are used in Economics and related fields, where advanced mathematics are often used for modeling and computation. We refer the reader to Abadir [4] for an extensive survey on their presence there. In Finance, the well-known Black-Scholes model now has its generalization to hypergeometric functions (Albanese et al. [91]).

\subsection{Random Matrices in Theoretical Physics}

Hypergeometric functions are frequently seen in theoretical physics and Appell's $F_{1}($. function is associated with several results related to the Shrodinger equation (see Exton [28]). It should be mentioned that the Theory of Random Matrices, developed independently in theoretical physics, has strong connections with matrix variate distributions in Mutivariate statistics. The various distributions associated with the eigenvalues of the Wishart matrix distribution were the connecting link between the two disciplines, and Wishart's [92] pioneering work on the distribution of the covariance matrix has been often cited in Physics. But the laws of Wigner, Tracy-Widom and Marcenko-Pastur developed there, have now found applications in Statistics (Johnstone [93]). 
On the other hand, $G$ and $H$-functions have numerous applications in Astrophysics, as can be seen in Chapter 5 of Mathai and Haubold [43].

\section{Conclusion}

The hypergeometric function and its generalizations have a place of choice in mathematics and its allied fields. We have given an overview of the roles this function plays across various domains and disciplines. In particular in Statistics, and Applied Statistics, its influence can be important in the years ahead and the statistician should be aware of its development in neighboring disciplines. We conclude this review by mentioning a reference bearing a special title [94], which clearly shows that hypergeometric functions can create an image which deeply affects the feelings of a researcher.

\section{Acknowledgements}

The first author wishes to thank colleagues at the Université de Moncton (Eric, Claude) and the Hochiminhcity University of Science (Trong, Bao, Nhat, Phong I and II, Dong, Hoa, Thin and Doan) for helping him understand and present various abstract mathematics concepts inherent to the latter part of this paper

\section{References}

[1] Bose, R.C. (1977) Early History of Multivariate Statistical Analysis. Multiv. In: Krishnaiah, P.R., Ed., Analysis IV, North-Holland, Amsterdam, 3-22.

[2] Encyclopedia of Statistical Sciences (2006) Hypergeometric Functions. Wiley on-line.

[3] Schlosser, M.J. (2013) Multiple Hypergeometric Series: Appell Series and Beyond. In: Schneider, C. and Blümlein, J., Eds., Computer Algebra in Quantum Field Theory: Integration, Summation and Special Functions, Springer, New York, 305-324.

[4] Abadir, M.K. (1999) An Introduction to Hypergeometric Functions for Economists. Econometric Reviews, 18, 287-330. http://dx.doi.org/10.1080/07474939908800447

[5] Wikipedia (2015) Hypergeometric Functions. Document on the Internet. https://en.wikipedia.org/wiki/Hypergeometric functions

[6] Encyclopedia of Mathematics (2007) Hypergeometric Functions. Vol. 4, Kluwer Acad.Pub., London.

[7] Ehrenpreis, L. (1991) Hypergeometric Functions. ICM-90 Satellite Conference Proceedings: Special Functions, Masaki Kashiwara, Tetsuji Miwa, Springer, 78-89.

[8] Cattani, E. (2006) Three Lectures on Hypergeometric Functions. Notes on the Internet. http://people.math.umass.edu/ cattani/hypergeom lectures.pdf

[9] Askey, R. (1979) Review of Special Functions of Applied Mathematics by B.C. Carlson. SIAM Review, 21, 420-421. http://dx.doi.org/10.1137/1021080

[10] Carlson, B.C. (1977) Special Functions of Applied Mathematics. Academic Press, New York.

[11] Gelfand, I., Kapranov, M. and Zevelinsky, A. (1991) Hypergeometric Functions, Toric Varieties and Newton Polyhedra. ICM-90 Satellite Conference Proceedings: Special Functions, published by Masaki Kashiwara, Tetsuji Miwa, Springer, 104-121.

[12] Saito, M., Sturmfels, B. and Takayama, N. (2000) Grobner Deformations of Hypergeometric Differential Equations. Springer, New York. 
http://dx.doi.org/10.1007/978-3-662-04112-3

[13] James, A.T. (1968) Calculation of Zonal Polynomial Coefficients by Use of the LaplaceBeltrami Operator. The Annals of Mathematical Statistics, 39, 1711-1718.

[14] Constantine, A.G. and Muirhead, R. (1972) Partial Differential Equations for Hypergeometric Functions of Two Argument Matrices. J. Multiv. Anal, 3, 332-338. http://dx.doi.org/10.1016/0047-259X(72)90020-6

[15] Erdélyi, A., Magnus, W., Oberhettinger, F. and Tricomi, F.G (1955) Higher Transcendental Functions. Vol. III, McGraw-Hill Book Company, Inc., New York-Toronto-London.

[16] Slater, L. (1960) Confluent Hypergeometric Functions. Cambridge University Press, London.

[17] Slater, L. (1966) Generalized Hypergeometric Functions. Cambridge University Press, Cambridge, UK.

[18] Bailey, W.N. (1935) Generalized Hypergeometric Series, Cambridge Tracts in Mathematics and Mathematical Physics 32. Cambridge University Press, London.

[19] Lebedev, N.N. (1972) Special Functions and their Applications. Dover, New York.

[20] Hogg, R.V. and Craig, A.T. (1995) Introduction to Mathematical Statistics. 5th Edition, Prentice-Hall, New York.

[21] Appell, P. and Kampé de Fériet, J. (1926) Fonctions Hypergéométriques, Hypershériques, Polynomes d'Hermite, Gauthier-Villars, Paris.

[22] Kummer, E.E. (1836) Uber die Hypergeometrische Reihe. Journal für die reine und angewandte Mathematik, 15, 39-83. http://dx.doi.org/10.1515/crll.1836.15.39

[23] Mathai, A.M. and Saxena, R.K. (1973) Generalized Hypergeometric Functions with Applications in Statistics and Physical Sciences. Springer-Verlag, New York. http://dx.doi.org/10.1007/BFb0060468

[24] Mainardi, F. and Pagnini, G. (2003) Salvatore Pincherle: The Pioneer of the Mellin-Barnes Integrals. Journal of Computational and Applied Mathematics, 153, 331-342. http://dx.doi.org/10.1016/S0377-0427(02)00609-X

[25] Olsson, O.M. (1966) Analytic Continuation of Hypergeometric Functions. Journal of Mathematical Physics, 7, 702-710. http://dx.doi.org/10.1063/1.1704985

[26] Springer, M. (1984) The Algebra of Random Variables. Wiley, New York.

[27] Rathie, P.N. (1989) Generalized Hypergeometric Functions and Exact Distributions of Test Statistics. American Journal of Mathematical and Management Sciences, 9, 155-172. http://dx.doi.org/10.1080/01966324.1989.10737258

[28] Exton, H. (1976) Multiple Hypergeometric Functions and Applications. Ellis Horwood, Chichester, New York.

[29] Srivastava H.M. and Karllson, M. (1977) Multiple Hypergeometric Functions, Ellis Horwood, London.

[30] Muirhead, R. (2005) Aspects of Multivariate Statistical Theory. Wiley, New York.

[31] Buschman, R.G. (1978) H-Functions of Two Variables. Indian Journal of Mathematics, 20, 105-116.

[32] Nguyen, T.H. and Yakubovich, S.B. (1992) The Double Mellin-Barnes Type Integrals and their Applications to Convolution Theory. World Scientific, Singapore.

[33] Mathai, A.M. and Saxena, R.K. (1978) The H-Function with Applications in Statistics and Other Disciplines. Halsted, New Delhi.

[34] Mathai, A.M., Saxena, R.K. and Haubold, H.J. (2010) The H-Function, Theory and Appli- 
cations. Springer, New York.

[35] Higham, N.J. (2008) Functions of Matrices, Theory and Computation. SIAM Publication, Philadelphia.

[36] Pham-Gia, T. and Turkkan, N. (2011) Distribution of the Ratio, from Random Variable to Random Matrix. Open Journal of Statistics, 1, 93-104. http://dx.doi.org/10.4236/ojs.2011.12011

[37] Gupta, A.K. and Nagar, D.K. (2000) Matrix Variate Distributions. Chapman and Hall/CRC, Boca Raton.

[38] Herz, C. (1955) Bessel Functions of Matrix Argument. Annals of Mathematics, 61, 474-523. http://dx.doi.org/10.2307/1969810

[39] Gross, K.I. and Richards, D.S.P. (1987) Special Functions of Matrix Argument I: Algebraic Induction, Zonal Polynomials, and Hypergeometric Functions. Transactions of the American Mathematical Society, 301, 781-811. http://dx.doi.org/10.2307/2000670

[40] Kates, L.K. (1980) Zonal Polynomials. PhD Dissertation, Princeton University, Princeton.

[41] Saw, J.G. (1977) Zonal Polynomials, an Alternative Approach. Journal of Multivariate Analysis, 7, 461-467. http://dx.doi.org/10.1016/0047-259X(77)90086-0

[42] Takemura, A. (1984) Zonal Polynomials. ISM Lecture Notes-Monograph Series, Vol. 4.

[43] Bekker, A., Roux, J.J.J. and Arashi, M. (2011) Exact Nonnull Distribution of Wilks's Statistic: Ratio and Product of Independent Components. Journal of Multivariate Analysis, 109, 73-87. http://dx.doi.org/10.1016/j.jmva.2012.02.016

[44] Mathai, A.M. (1991) Special Functions of Matrix Arguments and Statistical Distributions. Indian Journal of Pure and Applied Mathematics, 22, 887-903.

[45] Mathai, A.M. and Haubold, H.J. (2008) Special Functions for Applied Scientists. Springer Science + Business Media, LLC, Berlin.

[46] Mathai, A.M. and Pederzoli, G. (1995) Hypergeometric Functions of Many Matrix Variables and Distributions of Generalized Quadratic Forms. Amer. Journ. of Matl and Manag. Sciences, 15, 343-354.

[47] Pearson, J. (2009) Computation of Hypergeometric Functions. MSc Thesis, Oxford University, Document on the Internet.

[48] Adamchik, V. (1995) The Evaluation of Integrals of Bessel Functions via G-Function Densities. Journal of Computational and Applied mathematics, 64, 283-290. http://dx.doi.org/10.1016/0377-0427(95)00153-0

[49] Gaskell, K. (1994) A Definite Integration Interface for REDUCE. Presentation on the Internet. https://www.scribd.com/.../A-Definite-Integration-Interface-for-REDUCE

[50] Gutiérrez, R., Rodriguez, J. and Sáez, A.J. (2000) Approximation of Hypergeometric Functions with Matricial Argument through Their Development in Series of Zonal Polynomials. Electronic Transactions on Numerical Analysis, 11, 121-130.

[51] Koev, P. and Edelman, A. (2006) The Efficient Evaluation of the Hypergeometric Function of a Matrix Argument. Mathematics of Computation, 75, 833-846. http://dx.doi.org/10.1090/S0025-5718-06-01824-2

[52] Butler, W.R. and Wood, A.T.A. (2005) Laplace Approximation for Hypergeometric Functions of two Matrix Arguments. Journ of Multiv. Analysis, 94, 1-16.

[53] Baleanu, D., Diethelm, K., Scalas, E. and Trujillo, J.J. (2012) Fractional Calculus, Models and Numerical Methods. World Scientific, Singapore. http://dx.doi.org/10.1142/8180

[54] Ledoit, O. and Wolfe, M. (2012) Non-Linear Shrinkage Estimation of Large-Dimensional Covariance Matrices. Annals of Statistics, 40, 1024-1060. 


\section{http://dx.doi.org/10.1214/12-AOS989}

[55] Fujikoshi, Y. and Ulyanov, V.V. (2010) Multivariate Statistics: High-Dimensional and Large-Sample Approximations. Wiley, New York. http://dx.doi.org/10.1002/9780470539873

[56] Milgram, M.S. (2006) On Hypergeometric ${ }_{3} F_{2}(1)$, arXiv:math/0603096.

[57] Prudnikov, A.P., Brychkov, Y.A. and Marichev, O.I. (1990) Integrals and Series. Vol. 3, Gordon and Breach, New York.

[58] Hannah, J.P. (2013) Identities for the Gamma and Hypergeometric Functions: An Overview from Euler to the Present. MSc. Thesis, School of Mathematics, University of the Witwatersrand, Johannesburg.

[59] Kiryakova, V. (1997) All the Special Functions are Differintegrals of Elementary Functions. Journal of Physics A: Mathematical and General, 30, 5085-5113. http://dx.doi.org/10.1088/0305-4470/30/14/019

[60] Lavoie, J.L, Osler, T.J and Tremblay, R. (1974) Fractional Derivatives and Special Functions. SIAM Reviews, 18, 240-268. http://dx.doi.org/10.1137/1018042

[61] Pham-Gia, T. (1976) Saturation and an Iteration Construction Process. Journal of Approximation Theory, 16, 234-242. http://dx.doi.org/10.1016/0021-9045(76)90051-4

[62] Mathai, A.M. and Haubold, H.J. (2011) Matrix Variate Statistical Distributions and Fractional Calculus. Fractional Calculus and Applied Analysis, 14, 138-155. http://dx.doi.org/10.2478/s13540-011-0010-Z

[63] Giri, N. (1996) Invariance in Statistical Inference. World Scientific Publishing, Singapore.

[64] Wijsman, R.A. (1990) Invariant Measures on Groups and their Use in Statistics. IMS Lecture Notes Monograph Series, Vol. 14, Hayward, California, USA.

[65] Wasson, R.D. and Gilmore, R. (2013) Classical Special Functions and Lie Groups. Drexel University, Pa, USA, Note on the Internet. http://dx.doi.org/10.1137/0503004

[66] Miller Jr., W. (1972) Lie Theory and Generalized Hypergeometric Functions. SIAM Journal on Mathematical Analysis, 3, 31-44.

[67] Miller Jr, W. (1974) Lie Theory and Meijer's G-Function. SIAM Journal on Mathematical Analysis, 5, 309-318. http://dx.doi.org/10.1137/0505034

[68] Fletcher, P.T., Lu, C. and Joshi, S. (2003) Statistics of Shape via Principal Component Analysis on Lie Groups. CVPR 2003 Proceedings, 1, 95-101.

[69] Dickey, J.M. (1983) Multiple Hypergeometric Functions: Probabilistic Interpretations and Statistical Uses. Journal of the American Statistical Association, 78, 628-637. http://dx.doi.org/10.1080/01621459.1983.10478022

[70] Carlson, B.C (2005) Jacobian Elliptic Functions as Inverse of an Integral. Journal of Computational and Applied Mathematics, 174, 355-359. http://dx.doi.org/10.1016/j.cam.2004.05.001

[71] Johnson, N. and Kotz, S. (1969) Discrete Distributions. Wiley, New York.

[72] Johnson, N., Kotz, S. and Balakhrisnan, N. (1997) Discrete Multivariate Distributions. Wiley, New York.

[73] Pham-Gia, T. and Turkkan, N. (1994) Reliability of a Standby System. IEEE Trans on Reliability, 43, 71-75. http://dx.doi.org/10.1109/24.285114

[74] Pham-Gia, T. (2000) Distribution of the Ratios of Beta Variables. Communications in Statistics - Theory and Methods, 29, 2693-2715. http://dx.doi.org/10.1080/03610920008832632

[75] Pham-Gia, T. (2008) Exact Expression of the Distribution of Wilks Statistic. Journal of Multivariate Analysis, 99, 1698-1716. http://dx.doi.org/10.1016/j.jmva.2008.01.021 
[76] Pham-Gia, T. and Choulakian, V. (2014) Distribution of the Sample Correlation Matrix and Applications. Open Journal of Statistics, 4, 330-344.

http://dx.doi.org/10.4236/ojs.2014.45033

[77] Farrell, R.H. (1985) Multivariate Calculations. Springer, New York. http://dx.doi.org/10.1007/978-1-4613-8528-8

[78] Pillai, K.C.S. (1977) Distributions of Characteristic Roots in Multivariate Analysis. Part II. Non-Null Distributions. Canadian Journal of Statistics, 5, 1-62. http://dx.doi.org/10.2307/3315084

[79] Olkin, I. and Rubin, H. (1964) Multivariate Beta Distributions and Independence Properties of Wishart Distributions. The Annals of Mathematical Statistics, 35, 261-269. http://dx.doi.org/10.1214/aoms/1177703748

[80] Gross, K.I. and Richards, D.S.P. (2009) On the Role of the Hypergeometric Functions with Matrix Argument in the Age of Electronic Communications. Presentation on the Internet. http://www.math.nist.gov/ DLozier/SF21/SF21slides/Richards.pdf

[81] Gupta, A.K. and Nadarajah, S. (2004) Handbook of Beta Distribution and Applications. Marcel Dekker, New York.

[82] Pham-Gia, T. and Turkkan, N. (1993) Bayesian Analysis of the Difference of Two Proportions. Communications in Statistics-Theory and Methods, 22, 1755-1771. http://dx.doi.org/10.1080/03610929308831114

[83] Chiani, M., Win, M.Z. and Shin, H. (2010) MIMO Networks: the Effects of Interference. IEEE Transactions on Information Theory, 56, 336-350. http://dx.doi.org/10.1109/TIT.2009.2034810

[84] Tulino, A.M. and Verdu, S. (2004) Random Matrices and Wireless Communications. Now Publishers Inc., Boston, Delft.

[85] Wolfram, S. (2005) The History and Future of Special Functions. Talk Given at the Wolfram Technology Conference 2005, Champaign.

[86] Aomoto, K. and Kita, M. (2011) Theory of Hypergeometric Functions. Springer, New York. http://dx.doi.org/10.1007/978-4-431-53938-4

[87] Beukers, F. (2014) Hypergeometric Functions, How Special Are They? Notices of the AMS, 61, 48-56. http://dx.doi.org/10.1090/noti1065

[88] Varchenko, A. (1990) Multidimensional Hypergeometric Functions in Conformal Field Theory, Algebraic K-Theory, Algebraic Geometry. Proceedings of the International Congress of Mathematicians, Kyoto, 281-300.

[89] Beukers, F. and Heckman, G. (1989) Monodromy for the Hypergeometric Function ${ }_{n} F_{n-1}$. Inventiones Mathematicae, 95, 325-354. http://dx.doi.org/10.1007/BF01393900

[90] Perelomov, A.M. (2004) Hypergeometric Solutions of Some Algebraic Equations. Theoretical and Mathematical Physics, 140, 895-904. http://dx.doi.org/10.1023/B:TAMP.0000033027.63336.80

[91] Albanese, C., Campolieti, G., Carr, P. and Lipton, A. (2005) Black-Scholes Goes Hypergeometric. Risk, December 2001, 99-103.

[92] Wishart, J. (1928) The Generalised Product Moment Distribution in Samples from a Normal, Multivariate Population. Biometrika, 20, 32-52.

[93] Johnstone, I. (2001) On the Distribution of the Largest Eigenvalue in Principal Components Analysis. The Annals of Statistics, 29, 295-327. http://dx.doi.org/10.1214/aos/1009210544

[94] Yoshida, M. (1997) Hypergeometric Functions, My Love. Aspects of Mathematics Series, Volume 32, Springer, New York. http://dx.doi.org/10.1007/978-3-322-90166-8 
Submit or recommend next manuscript to SCIRP and we will provide best service for you:

Accepting pre-submission inquiries through Email, Facebook, LinkedIn, Twitter, etc. A wide selection of journals (inclusive of 9 subjects, more than 200 journals)

Providing 24-hour high-quality service

User-friendly online submission system

Fair and swift peer-review system

Efficient typesetting and proofreading procedure

Display of the result of downloads and visits, as well as the number of cited articles

Maximum dissemination of your research work

Submit your manuscript at: http://papersubmission.scirp.org/

Or contact ojs@scirp.org 